\title{
Why are testing rates so low in sub-Saharan Africa? Misconceptions and strategic behaviors
}

\author{
Olivier Sterck ${ }^{1}$
}

\begin{abstract}
Voluntary testing and counseling (VTC) is a popular method for fighting the HIV/AIDS epidemic. The purpose of VTC is to reduce the incidence of the virus in a twofold manner. First, testing provides access to health care and antiretroviral therapies that diminish the transmission rate of the virus. Second, counseling encourages safer behavior for not only individuals who test HIV-negative and wish to avoid HIV/AIDS infection but also altruistic individuals who test HIV-positive and wish to protect their partners from becoming infected by HIV. Surprisingly, DHS surveys that were conducted in sub-Saharan Africa provide empirical evidence that testing services are underutilized. Moreover, it is rare for both partners in a couple to be tested for HIV. This paper proposes a theoretical model that indicates how misperceptions about the HIV/AIDS virus may explain these puzzles. More specifically, this study demonstrates that individuals who are at risk of HIV infection may act strategically to avoid the cost of testing if they overestimate the risk of HIV transmission or believe that health care is not required if HIV is asymptomatic. The correction of false beliefs and the promotion of self-testing are expected to increase HIV testing rates.
\end{abstract}

Keywords: HIV/AIDS, transmission rate, testing, prevention, risk perception JEL Classification: I10, I15, I18, O12

\section{Introduction}

In 2009, an estimated 33.3 million people were living with HIV (Who, UNAIDS \& UNICEF, 2010). Although increasing quantities of resources have been dedicated to limiting the spread of the HIV/AIDS epidemic, UNAIDS reports that approximately 2.6 million people became infected with HIV in 2009. Voluntary testing and counseling (VTC) is regarded as one of the most powerful policy tools that are available for fighting this epidemic. Indeed, testing may reduce the spread of HIV/AIDS in two ways. First, testing provides individuals with access to health care and antiretroviral therapies (ARV), which sharply reduce the transmission rate of HIV by diminishing the viral load in the blood (Castilla et al., 2005). Second, the receipt of an HIV test result may foster safer behavior among individuals who are altruistic and tested positive and individuals who are fearful and tested negative. From empirical evidence, it is unclear whether testing limits the spread of HIV or encourages risky behavior (Thornton, 2008; Delavande and Kohler, 2009; Gong, 2010).

The sixth United Nations Millennium Development Goal is to "achieve, by 2010, universal access to treatment for HIV/AIDS for all those who need it". Nowadays, both antiretroviral therapies and voluntary testing and counseling services are available for free in a large majority of African countries. Despite this increase in access to VTC, the use of these services remains low. Furthermore, individuals who choose to be tested for HIV often do not return to receive their results (Obermeyer and Osborn, 2007).

For instance, in Lesotho, between 58 and $83 \%$ of individuals with advanced HIV infections ${ }^{2}$ received antiretroviral therapies in 2006 (according to WHO statistics). Despite this widespread access to HIV treatment, only $1.98 \%$ of the

\footnotetext{
Email address: olivier.sterck@economics . ox .ac .uk (Olivier Sterck)

${ }^{1}$ The author would like to thank the ARC project 09/14-018 on "sustainability" (French speaking community of Belgium) for its financial support. Any opinions, findings, and conclusions or recommendations expressed in this publication are those of the author.

${ }^{2} \mathrm{An}$ advanced HIV infection corresponds to a CD4 count that is at or below 350 cells $/ \mathrm{mm}^{3}$ (in accordance with WHO guidelines that were enacted prior to 2009).
} 
total number of couples that responded to a DHS survey of Lesotho were couples in which both partners had been tested for HIV and received the test results (table 1). For $13.99 \%$ of the responding couples, only one partner of the couple was tested and received the results of the test. The remaining $84.04 \%$ of couples had received no test results (either because neither member of the couple had been tested or because one or both members of the couple had been tested but did not obtain the test results). This result is particularly surprising because in $79.74 \%$ of the couples that had never been tested for HIV, at least one partner wanted to be tested. Moreover, in $43.46 \%$ of the couples that had never been tested for HIV, both members of the couple wanted to be tested.

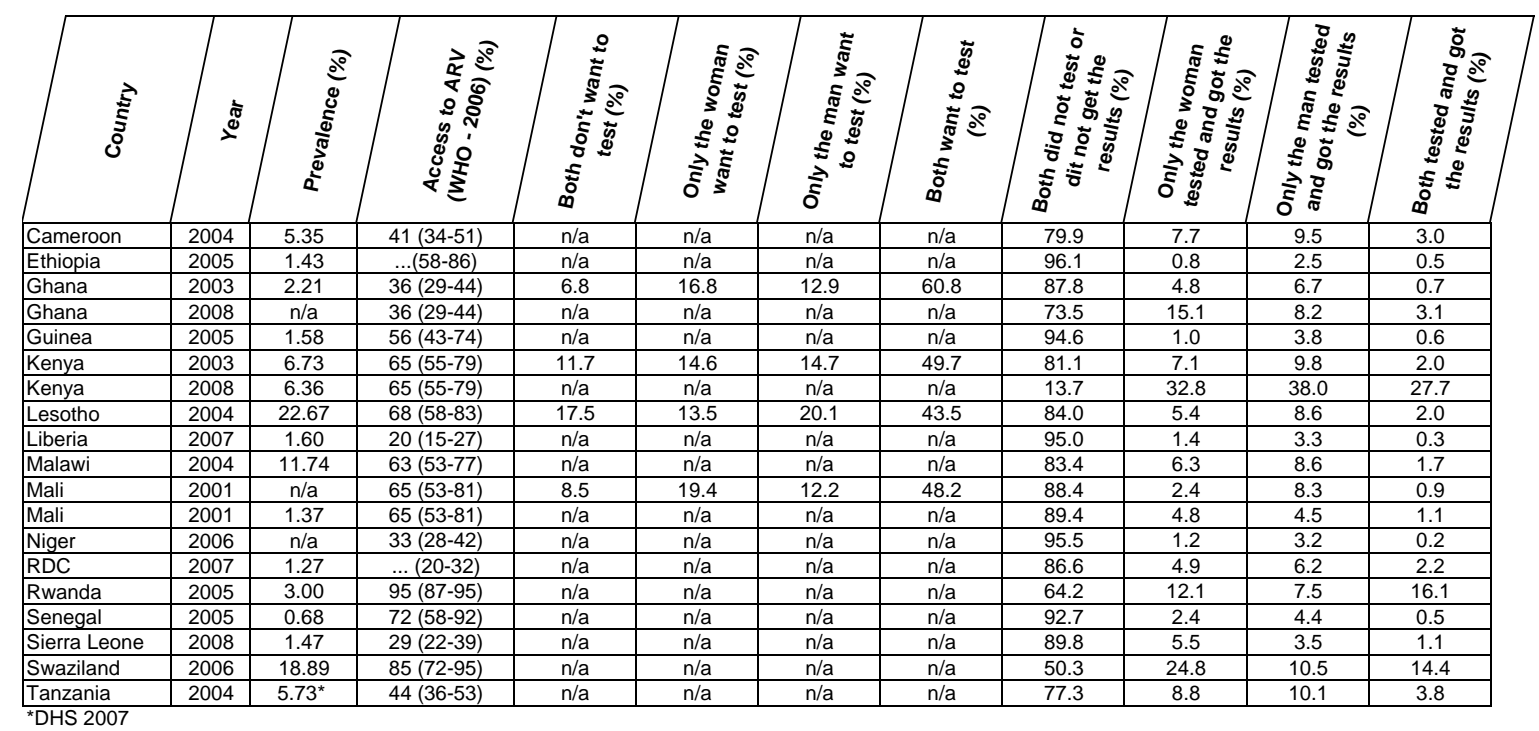

Table 1: Testing patterns in sub-Saharan Africa (DHS).

A similar pattern of behavior is observed in other sub-Saharan countries. More specifically, two observations can be made from table 1. First, despite the high prevalence of HIV in sub-Saharan nations and the fact that residents of these nations enjoy increasing levels of access to antiretroviral therapies, testing rates remain astonishingly low in these countries. This phenomenon is rather surprising given that the intention to test for HIV is generally high. As a consequence, the majority of infected individuals in sub-Saharan Africa remain unaware of their serostatus (Whо, UnAids \& Unicef, 2009). Second, table 1 indicates that the proportion of couples in which both partners have been tested for HIV is low relative to the proportion of couples in which only one of the partners has been tested. As will be discussed, these two observations are difficult to reconcile with existing theoretical models that examine the decision to test for HIV. The objective of this paper is to demonstrate that both strategic considerations by couples and misconceptions about the situations in which HIV treatment is required may explain the low testing rates in sub-Saharan Africa, particularly within the context of steady relationships.

In the existing theoretical models, testing either represents a required signal for obtaining access to the risky sex market or a strategy that is used by altruistic individuals to avoid infecting their partners (Philipson and Posner, 1993, 1995; Boozer and Philipson, 2000). Given the high prevalence of HIV in sub-Saharan Africa, these models predict that high testing rates should be observed in this region because individuals either have to signal that they are uninfected or wish to protect their sexual partners. These predictions are not supported by the observed patterns of HIV testing.

To explain the low testing rates in sub-Saharan Africa, another strand of the literature emphasizes the importance of both objective and subjective costs that are associated with testing and treatment (Gersovitz, 2011). Access to 
HIV treatment is often reported to be a determinant of an individual's willingness to be tested (Creek et al., 2006; Kaler and Watkins, 2010). In accordance with this perspective, empirical evidence has revealed that the decision to test for HIV is highly responsive to both the price of antiretroviral drugs (Kautz, 2008) and the direct costs of testing (Thornton, 2008; Thielman et al., 2006; Wolff et al., 2005). In addition to the objective costs and benefits of testing, many studies have emphasized that individuals fear HIV testing for subjective reasons (Gersovitz and Hammer, 2003; Gersovitz, 2011). In particular, the fear of receiving an positive result has commonly been reported to be a reason that individuals avoid testing (Obermeyer and Osborn, 2007; Bwambale et al., 2008; Kaler and Watkins, 2010). Many studies have also indicated that individuals do not undergo HIV testing because they fear violent reactions, potential separations from their partners and social stigmas (Obermeyer and Osborn, 2007). The fear of negative outcomes after testing partially explains the low testing rates in sub-Saharan Africa. This fear is expected to be present at higher levels for women and for other marginalized groups. Therefore, HIV testing is expected to be more frequent among men; however, this result is not consistently observed in table 1.

In accordance with the analysis of Gersovitz and Hammer (2003), this paper argues that misconceptions about HIV/AIDS may induce strategic behavior with respect to individual choices about testing. A model of the decision to test for HIV will be constructed; in this model, testing is a costly process but provides access to health care for individuals who test positive. To avoid testing costs, which may be high because of subjective considerations (such as fear and the social stigmas that are associated with being HIV-positive), partners may act strategically and postpone HIV testing. This paper focuses on two mechanisms that may explain why HIV testing rates are lower than expected.

The first of these mechanisms is related to the observability of a partner's test results and a biased knowledge regarding the HIV transmission rate for each act of risky behavior. Taken together, these two subjective variables may explain why partners fail to test for HIV in couples. The intuition is as follows. Let us imagine that a person $i$ has already engaged in unprotected sexual encounters with his regular partner $j$ and believes that the transmission rate of HIV is high ${ }^{3}$. In this situation, $i$ expects to have the same serostatus as his partner; in particular, $i$ believes that if either $i$ or $j$ was infected with HIV prior to their relationship, then HIV is expected to have been transmitted during the unprotected sexual encounters between $i$ and $j$. Therefore, if $i$ believes that his partner will be tested and that he will observe the test result, he may act strategically to avoid the cost of testing. In this case, he will test for HIV only if his partner is observed to be HIV-positive.

The incentive to be strategic and postpone testing is amplified by the fact that most individuals overestimate the infectivity of HIV. The transmission rate of HIV during each unprotected sexual act is generally lower than $1 \%$ (Boily et al., 2009). However, individuals who are asked about the probability of becoming infected as a result of one unprotected sexual act with an HIV-positive partner typically greatly overestimate this probability. For instance, the average expected HIV transmission rate is $86.8 \%$ in rural Malawi (Delavande and Kohler, 2009) and 81.4\% in Burundi (Sterck, 2012a). Similar misconceptions about the infectivity of HIV are also common in developed countries (Linville et al., 1993; Pinkerton et al., 2000; Knauper and Kornik, 2004). Because individuals overestimate the per-act transmission rate of HIV, they are inclined to believe that serodiscordance ${ }^{4}$ is impossible within established relationships that feature frequent unprotected sexual encounters. Because serodiscordance is thought to be unlikely, an observation of a partner's test result is expected to be "fully informative"; therefore, only one partner from each couple is expected to be tested for HIV. This reasoning indicates that it may be possible to increase testing rates by disclosing that serodiscordance is possible and that the per-act transmission rate of HIV is much lower than most individuals would predict.

The second mechanism for explaining unexpectedly low rates of HIV testing relates to the belief that HIV treatment is not required if the disease is asymptomatic. The intuitive presentation of this explanation may be expressed as follows. If an individual $i$ believes that treatment is required only if AIDS symptoms are observable, he will postpone testing and test for HIV only after the onset of AIDS symptoms. This strategic behavior may explain the high rates of

\footnotetext{
${ }^{3}$ For the ease of the exposition, we assume that the decision-maker $i$ is a man and that the partner $j$ a woman. However, the findings of this paper hold for both heterosexual and homosexual couples.

${ }^{4} \mathrm{~A}$ Serodiscordant relationship is one in which one partner is HIV positive and the other is HIV negative.
} 
late testing among HIV-positive individuals (Whо, UnAIDs \& UNICEF, 2009). This reasoning also provides a rationale for why testing rates in sub-Saharan Africa remain low despite this region's high prevalence of HIV and its recent improvements in access to treatment.

In reality, the early diagnosis of HIV infection is a critical aspect of maximizing the effectiveness of treatment, decreasing the cost of HIV-related medical care and reducing the infectivity of HIV (Girardi et al., 2007). Therefore, the dissemination of the fact that the effectiveness of treatments diminishes as an HIV infection progresses may increase early testing, reduce incidence and improve health outcomes for HIV-positive individuals.

To the best of my knowledge, this paper is the first to formally discuss how strategic behavior may reduce individuals' incentives to be tested for HIV. The two proposed mechanisms provide an explanation for the low testing rates in sub-Saharan Africa and for the low proportion of couples in which both partners had been tested. This paper complements the existing literature on the decision to test for HIV by studying how misconceptions about HIV/AIDS may reduce the incentives to test and instead encourage strategic behavior (see Gersovitz (2011) for a recent review that addresses HIV testing). This paper is also related to the large literature on behavioral changes that occur after testing (Boozer and Philipson, 2000; Thornton, 2008; Delavande and Kohler, 2009; Gong, 2010). Theoretically, these studies indicate that only individuals who are surprised by their test results should change their behaviors. The direction of this change depends on the relative importance of expectation revisions and altruism. Empirical analyses of these changes have produced contradictory results. Finally, this paper is also related to the literature stream that addresses strategic behaviors with respect to misconceptions about HIV/AIDS (O'Donoghue and Rabin, 2001; Gersovitz and Hammer, 2003; Sterck, 2012a). This literature reveals that individuals may be prone to fatalism and may rationally engage in risky behavior if they overestimate the infectivity or the prevalence of HIV.

This paper is organized as follows. The second section develops a theoretical model in which HIV testing provides access to treatments. This framework predicts that individuals and couples may act strategically if they overestimate the transmission rate of HIV and believe that a partner's test result is observable. In the third section, the definition of observability is modified to study how beliefs about when treatment is necessary may impact HIV testing behavior. In accordance with observed patterns, this modified framework predicts that partners will postpone HIV testing if they believe that antiretroviral therapies are not required when HIV is asymptomatic. The fourth section compares the long-run predictions of the developed models with empirical evidence from the literature. The last section concludes and discusses the policy implications of the study.

\section{A model of HIV testing}

\subsection{The construction of the model}

An individual $i$ is in a couple with another individual $j^{5}$. Both $i$ and $j$ must choose whether to be tested for HIV. Without loss of generality, we focus on $i$ 's decision process. The sequence of this decision process is depicted in figure 1 .

Prior to the first testing decision, $i$ has participated in $n \geq 0$ risky sexual encounters with $j$. Throughout the paper, the cases of $n=0$ and $n \geq 1$ will be distinguished and compared. There are several reasons why couples may engage in unprotected sex without first testing for HIV. First, many couples may have engaged in unprotected sexual encounters before they have obtained access to HIV testing. This situation is particularly prevalent in remote rural areas, where HIV testing has become progressively more available over the course of the past decade. Second, even where testing facilities were accessible, individuals may have been reluctant to be tested in the past if HIV treatments were not available at a reasonable cost. Now that the access to antiretroviral therapies is more widespread, people may be willing to test in order to have access to treatment. Third, even if individuals are willing to avoid HIV infection and if testing facilities are available for free, they may indulge in risky sex before testing because of the "heat of

\footnotetext{
${ }^{5}$ For the purposes of this explanation, it is assumed that $i$ is a man and that $j$ is a woman; however, the findings of this paper hold for both heterosexual and homosexual couples.
} 
the moment", condom leakage or misconceptions about condoms (Flood, 2003; Chimbiri, 2007; Moore and Oppong, 2007; Maticka-Tyndale and Kyeremeh, 2010). In line with this, Sanders et al. (2012) compiled an interesting review of frequent errors in condom use. Then, after few unprotected sexual encounters, partners may abandon condom usage because of either habituation or fatalism (Rhodes, 1997; Flood, 2003; Sterck, 2012a). This is consistent with empirical evidence showing that condom use is well accepted for encounters with casual sexual partners but generally abandoned with regular partners (Meekers et al., 2003; de Walque, 2006; Moore and Oppong, 2007). Therefore, even if partners are willing to be tested for HIV before engaging in risky sex, they may fail to do so in practice. Finally, the HIV virus is characterized by a "window period" of approximately three months; during this period, HIV tests are unable to detect HIV antibodies, which serve as indicators of an HIV infection (Gersovitz, 2011). Either the "heat of the moment" or condom leakage may cause new partners to engage in risky sex prior to the end of this window period (in other words, before HIV testing is fully informative).

We use $p_{i i}$ to represent $i$ 's estimate of the probability that he has been infected with HIV prior to his relationship with $j$, and we use $p_{i j}$ to represent $i$ 's estimate of the probability that $j$ was infected at the beginning of the relationship between $i$ and $j$. The expected transmission rate of HIV per sexual act is denoted by $\beta$. The probabilities $p_{i i}$ and $p_{i j}$ are assumed to be strictly increasing and concave in $\beta$; these probabilities are equal to 0 if $\beta=0^{6}$. It is assumed that the expected probability $P_{n}$ that the HIV virus was transmitted during the course of the $n$ sexual encounters between $i$ and $j$ if $j$ was initially infected and $i$ was initially uninfected follows the binomial law ${ }^{7}: P_{n}=1-(1-\beta)^{n}$.

At time $t_{1}, i$ and $j$ choose whether to be tested $\left(t_{1}^{k}=1\right.$ if the individual $k$ tests at $t_{1}$ for $\left.k=i, j\right)$. The test costs $C$. In this model, $C$ includes both the objective costs (such as the amount that is paid to the testing facility) and the subjective costs (indirect costs that relate to individuals' concerns about social stigma, the reactions of their partner and the prospect of learning their serostatus) of testing. If infected, testing gives access to health care facilities and antiretroviral therapy; this access is assigned a value of $H$. HIV induces a disutility $d$. It is assumed that health care is more valuable than the cost of testing: $0<C<H$. Given this assumption, an individual that is certainly infected with HIV will always choose to undergo HIV testing to obtain access to health care. Even with access to ARV therapies, individuals will prefer an HIV-negative status to an HIV-positive status: $0<H<d$.

Both $i$ and $j$ believe that a positive test result is observable by their partner with a probability of $q$. In section 2.2 , the model will be solved for $q=0$. Section 2.3 addresses the case in which $q=1$ and demonstrates that the expected observability of a partner's test result encourages strategic behavior ${ }^{8}$.

Several reasons may explain why a positive test result would be observable by the partner of a tested individual. First, learning of an HIV-positive status typically produces a psychological shock that may be difficult to conceal. Second, the treatment of HIV/AIDS involves frequent visits to health facilities, and antiretroviral therapies require the daily intake of medications. It may be difficult to avoid revealing the existence of these treatments to a partner, particularly given the material deprivation that is faced by many African households and the important side effects of ARV treatments. Third, individuals may believe that their partner is altruistic and would provide them with advice in cases of infection. In a meta-review, Medley et al. (2004) find that disclosure rates with respect to HIV test results vary substantially, ranging between 16.7 and $86 \%$. Fears of abandonment, discrimination, violence or accusations of infidelity are frequently emphasized as reasons that may justify the decision to remain secretive regarding HIV test results (Medley et al., 2004).

If either $i$ or $j$ observes that his or her sexual partner is infected, then another HIV test may be performed at time $t_{2}$ to obtain access to ARV treatments $\left(t_{2}^{k}=1\right.$ if $k$ tests at $t_{2}$ for $\left.k=i, j\right)$. It is assumed that partners do not knowingly

\footnotetext{
${ }^{6} p_{i i}^{\prime}>0, p_{i i}^{\prime \prime} \leq 0, p_{i j}^{\prime}>0, p_{i j}^{\prime \prime} \leq 0$, and $p_{i i}(0)=p_{i j}(0)=0$. These assumptions are verified if the probability that the virus is transmitted follows a binomial law.

${ }^{7}$ Kaplan (1990) and Rottingen and Garnett (2002) argue that the binomial law is not consistent with empirical data regarding the transmissibility of HIV. However, it is assumed that individuals cannot distinguish the among different stages of HIV/AIDS and that they therefore act on the assumption of a constant per-act probability of transmission.

${ }^{8}$ The intermediate case of $0<q<1$, which is not examined in this study, leads to similar results with respect to strategic behavior (Sterck, 2012b).
} 
engage in risky sex with an infected person if they believe to be possibly uninfected. Therefore, if $i$ observes that $j$ is HIV-positive and if $i$ is not tested or is tested HIV-negative, it is assumed that, after the second testing decision in $t_{2}, i$ engages in $N$ unprotected encounters with a new partner whose expected probability of being infected is $p_{i j}$.

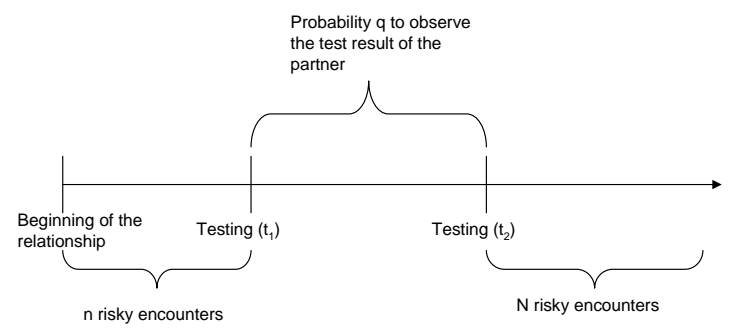

Figure 1: The sequence of the model.

An individual's behavioral choices after HIV testing primarily depend on the individual's altruistic and selfprotecting tendencies. The existing empirical studies that address behavioral changes after HIV testing provide conflicting results (Thornton, 2008; Delavande and Kohler, 2009; Gong, 2010). Because of these contradictory findings, it is assumed that behavior after testing is exogenous ${ }^{9}$. After the testing decisions at $t_{1}$ and $t_{2}, i$ and $j$ engage in $N \geq 0$ risky sexual encounters. These $N$ risky encounters provide a utility of $U_{N}$. If $j$ is infected and $i$ is uninfected at $t_{2}$, the expected probability $P_{N}$ that the virus is transmitted during the course of these $N$ encounters is equal to $P_{N}=1-(1-\beta)^{N}$

\subsection{Testing to obtain access to health care}

First, we solve the model with the assumption that the result of an HIV test will not observable by an individual's sexual partner (in other words, $q=0$ ).

If $i$ does not test at $t_{1}$, then he does not obtain $H$, the benefits from HIV-related health care. His expected lifetime utility is given by the difference between $U_{N}$, the utility of the $N$ risky encounters that occur after the testing decision, and the expected utility loss if he has AIDS:

$$
V\left(t_{1}^{i}=0\right)=U_{N}-\underbrace{\left[p_{i i}+p_{i j}\left(1-p_{i i}\right) P_{n}\right]}_{\text {i infected at } t_{1}} d-\underbrace{p_{i j}\left(1-p_{i i}\right)\left(1-P_{n}\right) P_{N}}_{\begin{array}{c}
i \text { infected during } \\
\text { the } N \text { encounters }
\end{array}} d .
$$

The term $\left[p_{i i}+p_{i j}\left(1-p_{i i}\right) P_{n}\right]$ is the expected probability that $i$ is infected at $t_{1}$. The term $p_{i j}\left(1-p_{i i}\right)\left(1-P_{n}\right) P_{N}$ is the expected probability that $i$ is not infected at $t_{1}$ but will become infected by $j$ throughout the course of the $\mathrm{N}$ risky encounters that occur after $i$ 's choice to not be tested at $t_{1}$.

By contrast, if $i$ chooses to test at $t_{1}$, he bears the testing cost, $C$, but obtains $H$, the benefits of access to HIVrelated health care, if the test reveals that $i$ is HIV-positive. The expected lifetime utility of $i$ may then be expressed as follows:

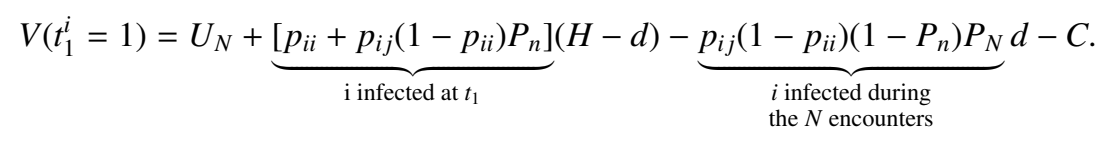

\footnotetext{
${ }^{9}$ The assumption that an individual behaves in altruistic or self-protective ways after the HIV test produces similar results (Sterck, 2012b).
} 
We define $A$ to represent the net benefit from being tested if a partner's test result is not observable, that is, the difference between equations (2) and (1):

$$
A=V\left(t_{1}^{i}=1\right)-V\left(t_{1}^{i}=0\right)=\underbrace{\left[p_{i i}+p_{i j}\left(1-p_{i i}\right) P_{n}\right]}_{\text {i infected at } t_{1}} H-C .
$$

In the above equation, $A$ is the difference between the expected utility of having access to health care if infected and the testing cost. The individual $i$ will test if this difference is positive ${ }^{10}$. This reasoning is summarized in the following proposition.

Proposition 2.1. If an individual $i$ believes that his partner's test result is not observable, then he tests for HIV if:

$$
A \geq 0 \Leftrightarrow \underbrace{\left[p_{i i}+\left(1-p_{i i}\right) p_{i j} P_{n}\right]}_{\begin{array}{c}
\text { Propensity } \Theta_{\text {A }} \text { to test } \\
\text { at } t_{1} \text { for health care }
\end{array}} \geq \frac{C}{H} .
$$

Proof The proof is in the text.

The propensity $\Theta_{A}$ measures an individual's willingness to be tested for HIV. This formulation will be useful for graphically representing the decision of the individual $i$. In summary, an individual $i$ will choose to be tested for HIV if his probability of being infected at $t_{1}$ is high and if the benefit from HIV-related health care is high compared with the cost of testing. Thus, $i$ tests if $p_{i i}, p_{i j}, P_{n}$ and $H$ are high and if $C$ is low.

Within this framework, the overestimation of $\beta$, the per-act transmission rate of HIV, favors testing. Indeed, the higher the expected transmission rate, the higher the probability to be infected at $t_{1}$. This reasoning is summarized in the following corollary.

Corollary 2.2. The propensity $\Theta_{A}$ is an increasing function of the expected transmission rate per act $\beta$. Therefore, if the test results of the individual's partner are expected to be unobservable, then the individual's overestimation of the HIV transmission rate may promote his decision to be tested for HIV.

Proof The proof is in the appendix.

\subsection{Strategic behavior: the observability of a partner's test results}

Let us now assume that the individual $i$ believes that he will observe a positive test result if his partner tests for HIV at $t_{1}$ and receives an HIV-positive result. In this case, $i$ may also test at $t_{1}$ to benefit from health care and antiretroviral therapy. However, if $i$ expects his partner $j$ to undergo HIV testing, then $i$ 's incentive to be tested for HIV is lowered because $i$ may be opportunistic and seek to observe the test results from $j$. In fact, to avoid the testing cost $C, i$ may rely on the fact that his partner is expected to be tested at $t_{1}$; therefore, $i$ may only undergo testing at $t_{2}$ if he observes that his partner $j$ is HIV-positive.

Thus, the decision to test is strategic and depends on an individual's beliefs about his partner's testing decision. If $j$ is not expected to undergo HIV testing, then the condition for $i$ to test is not modified: under these circumstances, $i$ will test if the condition $A \geq 0$ is satisfied.

By contrast, if $j$ is expected to undergo HIV testing at $t_{1}$, then $i$ believes that he will observe the test result if it is positive. If $i$ and $j$ have already engaged in risky sex before the time of the testing decision $(n>0)$, then $i$ 's observation of his partner's HIV test result provides him with information about his own serostatus: if $j$ is observed to be HIV-positive, $i$ will infer that he is likely to be infected because $i$ and $j$ have already engaged in unprotected sex.

\footnotetext{
${ }^{10} \mathrm{We}$ assume that $i$ chooses to be tested if he is indifferent.
} 
This phenomenon will be particularly prevalent if $\beta$, the expected transmission rate of HIV, is overestimated. Indeed, if $\beta$ is near 1 and if $n>0$, then the probability $P_{n}$ will be near 1 , and partners will believe that they share the same serostatus.

Therefore, to avoid testing costs, the individual $i$ may be tempted to free ride at $t_{1}$ and observe the HIV test result of his partner. If $j$ is observed to be HIV-positive, $i$ will undergo HIV testing at $t_{2}$ to obtain access to health care and antiretroviral therapy. If $j$ is HIV-negative, then $i$ will have saved $C$, the testing cost.

By contrast, if $i$ and $j$ never engaged in risky behavior $(n=0)$, then the observation that $j$ is HIV-positive does not provide any information about $i$ 's serostatus. In this situation, it is assumed that $i$ will not wish to engage in risky sex with his infected partner, and the $N$ unprotected sexual acts will involve a new partner whose expected probability of being infected with HIV is also equal to $p_{i j}$. This idea is formalized in the following proposition.

Proposition 2.3. If $j$ is not expected to test or if $i$ and $j$ have never engaged in unprotected sex $(n=0)$, then the individual $i$ tests for $H I V$ at $t_{1}$ if $A \geq 0$.

On the contrary, if $j$ is expected to test at $t_{1}$ and if $i$ and $j$ have had $n>0$ unprotected encounters before $t_{1}$, then the incentive for $i$ to be tested at $t_{1}$ is reduced by opportunism. In this case, i tests at $t_{1}$ if $A-\Omega \geq 0$, where the opportunism $\Omega$ is defined as follows:

$$
\Omega=\underbrace{\left[p_{i i} p_{i j}+p_{i i}\left(1-p_{i j}\right) P_{n}+\left(1-p_{i i}\right) p_{i j} P_{n}\right]}_{\text {both } \text { i and } j \text { are infected at } t_{1}} H-\underbrace{\left[p_{i j}+p_{i i}\left(1-p_{i j}\right) P_{n}\right]}_{j \text { is infected at } t_{1}} C .
$$

If $i$ does not undergo HIV testing at $t_{1}$, he will undergo HIV testing at $t_{2}$ if he observes that his partner is HIV-positive and if $\Omega \geq 0$.

Proof The proof is in the appendix.

Opportunism $\Omega$ is composed of two terms. The first term represents the expected health gain from testing at $t_{2}$ if an individual's partner has tested positive for HIV: if $i$ is opportunistic, he benefits from health care only if both he and his partner are infected. The second term is the cost of testing that $i$ bears if he tests at $t_{2}$ because he observes that his partner is infected. Opportunism $\Omega$ represents the fact that $i$ may obtain information about his own serostatus via the observation of $j$ 's HIV test results. $\Omega$ is increasing as $P_{n}$ grows. In particular, a greater probability $P_{n}$ that HIV was transmitted between $i$ and $j$ implies that a partner's test result is more informative and therefore creates greater incentives to engage in opportunistic testing behavior.

This reasoning has strong implications for the relationship between the decision to test for HIV and the overestimation of $\beta$, the per-act transmission rate for HIV. Corollary 2.2 should be adapted if a partner's HIV test results are expected to be observable. The intuition is as follows. On the one hand, if the expected transmission rate of HIV is overestimated $(\beta \approx 1)$, then $i$ and $j$ will falsely infer that serodiscordance is impossible if they have already engaged in risky sex and were faithful to each other. In this situation, the observation of a partner's test result is expected to be fully informative, and $i$ will be opportunistic if he believes that $j$ will test for HIV. This opportunistic reaction will be accentuated by $i$ 's overestimation of the transmission rate. Therefore, the dissemination of the fact that the transmission rate is lower than expected may increase the rates of HIV testing.

On the other hand, the expected probability that $i$ is HIV-positive at the time of the decision is increasing in the expected transmission rate $\beta$. Indeed, the more infectious HIV is expected to be, the higher is the expected probability to be infected. Therefore, if $\beta$ is very low, the expected probability of being HIV-positive at $t_{1}$ will be sharply reduced, and $i$ will wish to be tested neither at $t_{1}$ nor at $t_{2}$. 
The interplay of these two opposite effects implies that an intermediate expected transmission rate maximizes an individual's propensity to undergo HIV testing. As a consequence, corollary 2.2 should be revised for the situation in which $q=1$ and $j$ is expected to test. Corollary 2.2 revealed that the propensity $\Theta_{A}$ to test at $t_{1}$ if $q=0$ is an increasing function of the expected transmission rate. In this case, the overestimation of the transmission rate may favor testing. Let us define $\Theta_{A-\Omega}$ as the propensity to test at $t_{1}$ if the partner is expected to test and if the test result of the partner is expected to be observable:

$$
A-\Omega \geq 0 \Leftrightarrow \underbrace{\frac{p_{i i}\left(1-P_{n}\right)}{1-p_{i i} P_{n}}}_{\Theta_{A-\Omega}} \geq \frac{C}{H} .
$$

Similarly, the propensity $\Theta_{\Omega}$ to test at $t_{2}$ if the partner is observed to be HIV-positive is defined as follows:

$$
\Omega \geq 0 \Leftrightarrow \underbrace{\frac{p_{i i} p_{i j}+p_{i i}\left(1-p_{i j}\right) P_{n}+\left(1-p_{i i}\right) p_{i j} P_{n}}{p_{i j}+p_{i i}\left(1-p_{i j}\right) P_{n}}}_{\Theta_{\Omega}} \geq \frac{C}{H} .
$$

In contrast to $\Theta_{A}$, the propensity to undergo testing if $q=0$ which was shown to be increasing in $\beta$ (corollary 2.2), the propensity $\Theta_{A-\Omega}$ to test at $t_{1}$ if $q=1$ and $E\left(t_{j}\right)=1$ is an inverted U-shaped function of the expected transmission rate $\beta$. This result implies that the overestimation of the transmission rate $\beta$ may discourage partners from undergoing testing if they have already engaged in unprotected sex and if they expect to observe the test results for their partner. Corollary 2.4 summarizes these considerations and discusses their implications on HIV testing decisions.

Corollary 2.4. The propensity $\Theta_{A}$ is an increasing function of $\beta$, the expected per-act transmission rate. Therefore, if the HIV test results of an individual's partner are expected to be unobservable or if this partner is not expected to be tested for HIV, the overestimation of the transmission rate may foster testing.

By contrast, the propensity $\Theta_{A-\Omega}$ to test at $t_{1}$ is an inverted $U$-shaped function of $\beta$. As a consequence, if $j$ is expected to be tested for HIV at $t_{1}$ and if the test results are expected to be observable, the reduction of the expected transmission rate to an intermediate level $\beta^{*}$ would encourage $i$ to be tested at $t_{1}$ if there exists a $\beta^{*}<1$ such that $\Theta_{A-\Omega}\left(\beta^{*}\right) \geq 0$.

Proof The proof is in the appendix.

Proposition 2.3 and corollary 2.4 have strong policy implications. First, as expected, the model predicts that increased access to antiretroviral therapies and a reduction in both the objective and subjective costs of testing should increase the willingness of individuals to be tested for HIV.

Second, the dissemination of the fact that the transmission rate of HIV is lower than most individuals predict, which may occur through a prevention campaign, may reduce the feeling that serodiscordance is impossible in established sexual relationships and therefore encourage both members of couples to undergo HIV testing. With this in mind, preventive technologies aiming at reducing the transmission rate of HIV (such as antiretroviral therapies, circumcision, pre-exposure prophylaxis or microbicides) may reduce serodiscordance denial and strategic behavior, and thereby increase testing rates. However, this prediction should be regarded with caution because disclosures that the HIV transmission rate is low may foster riskier behavior. For example, Sterck (2012a) has demonstrated that reductions in the expected transmission rate are expected to increase condom use in established sexual relationships but also to increase the prevalence of risk-taking behaviors with casual sexual partners.

Finally, the fact that opportunism is increasing in $P_{n}$ suggests another channel through which testing rates could be increased. A reduction in $n$, that is, a reduction in the number of unprotected encounters that precede an HIV testing decision, is expected to reduce the general disbelief in serodiscordance between sexual partners and therefore reduce 
the propensity to engage in strategic behavior. This reduction could be accomplished by increasing the frequency of routine HIV testing, and by promoting home-based self-testing for HIV. This recent technological advance provides the potential to increase the accessibility of testing and to reduce the subjective cost of testing by shifting the HIV testing context from the medical sector to homes or other convenient locations (Gersovitz, 2011).

In the above discussion, we have assumed that the decision to undergo HIV testing is an individual choice. However, many couples discuss HIV testing and make decisions as a couple with respect to HIV. If the hypothesis of individual decision-making is relaxed, the intuition regarding the mechanisms underlying HIV testing decisions is unchanged, but the analysis becomes somewhat more complicated because of the presence of implicit altruism. Indeed, if a decision to be tested or not is reached in combination with a committed sexual partner and if the couple's utility function is maximized, then testing is valued not only because it provides access to HIV-related health care $H$, but also because it informs each partner of the other's serostatus and protects them from becoming infected by the virus. Within this modified framework, only one partner is expected to test in a committed couple if the transmission rate of HIV is overestimated and if partners have already engaged in unprotected sex. This extension to the theoretical framework is proposed in Appendix B.

Before performing simulations, let us discuss how asymmetric risk perceptions may affect the predictions of the model. It has been demonstrated that the per-act transmission rate of HIV is dependent on gender and that HIV prevalence is dependent on gender and age (Boily et al., 2009; Who, UnAIDs \& Unicef, 2009). On the one hand, note that the per-act expected transmission rate is overestimated to a similar extent by both males and females (Sterck, 2012a). Therefore, the assumption that individuals overestimate the infectivity of HIV appears to be satisfied for both genders. On the other hand, if the asymmetry of risk perception is related to the expected probabilities that each partner in a couple has been infected with HIV prior to the start of their relationship, strategic behavior is still expected to emerge if the transmission rate of HIV is significantly overestimated. Of course, if $n=0$, then the propensity to undergo HIV testing $\Omega_{A}$ is higher for the member of the couple who has the greatest risk of being infected. However, if $n \geq 1$ and $\beta \approx 1$, the two members of a couple will expect to have the same serostatus, even if one partner was expected to be much more likely to be infected at the beginning of their relationship.

\subsection{Simulations}

The predictions of propositions 2.1 and 2.3 are summarized in table 2. If $i$ believes that his partner will not undergo HIV testing or that the test results will not be observable, then he will decide to be tested at $t_{1}$ if $\Theta_{A} \geq \frac{C}{H}$. By contrast, if he believes that his partner will undergo testing at $t_{1}$, he will be tempted to be opportunistic and to postpone testing. In this situation, he undergoes testing at $t_{1}$ if $\Theta_{A-\Omega} \geq \frac{C}{H}$. Corollaries 2.2 and 2.4 revealed that the propensities $\Theta_{A}$ and $\Theta_{A-\Omega}$ are affected differently by the overestimation of the transmission rate. In this section, simulations are run to visualize these relationships and thereby evaluate how an individual's willingness to be tested for HIV is affected by changes in $P_{n}, \beta$ and $n$.

\begin{tabular}{|c|c|c|c|}
\hline \multirow{2}{*}{} & \multirow{2}{*}{ Test in $\boldsymbol{t}_{\mathbf{1}}$} & \multicolumn{2}{|c|}{ No test in $\boldsymbol{t}_{\mathbf{1}}$} \\
\cline { 3 - 4 } & & Test in $\boldsymbol{t}_{\mathbf{2}}$ & No test in $\boldsymbol{t}_{\mathbf{2}}$ \\
\hline $\boldsymbol{E}\left(\boldsymbol{t}_{\mathbf{1}}^{\mathbf{j}}\right)=\mathbf{0}$ or $\boldsymbol{q}=\mathbf{0}$ & $\Theta_{A} \geq \frac{C}{H}$ & \multicolumn{2}{|c|}{$\Theta_{A}<\frac{C}{H}$} \\
\hline $\boldsymbol{E}\left(\boldsymbol{t}_{\mathbf{1}}^{\boldsymbol{j}}\right)=\mathbf{1}$ and $\boldsymbol{q}=\mathbf{1}$ & $\Theta_{A-\Omega} \geq \frac{C}{H}$ & $\Theta_{\Omega} \geq \frac{C}{H}$ & $\Theta_{\Omega}<\frac{C}{H}$ \\
\hline
\end{tabular}

Table 2: Testing decision-making

Figures 2(a) and 2(b) graphically represent the propensities $\Theta_{A}, \Theta_{A-\Omega}$ and $\Theta_{\Omega}$ as a function of the probability $P_{n}$ that HIV was transmitted between $i$ and $j$ prior to an HIV testing decision ${ }^{11}$. The relative cost of testing $\frac{C}{H}$ is represented by a horizontal line.

\footnotetext{
${ }^{11}$ For these simulations, the expected probabilities $p_{i i}$ and $p_{i j}$ are fixed at an intermediate level: $p_{i i}=p_{i j}=5 \%$. The expected benefit from health care $H$ is normalized to 1 .
} 
In figure 2(a), the cost of testing is assumed to be low. In this situation, the propensity $\Theta_{A}$ is higher than the horizontal line $\frac{C}{H}$. Therefore, if test results are not observable or if the individual's partner is not expected to undergo HIV testing, the individual $i$ will test at $t_{1}$ because the cost of testing is low and because he believes that he is at risk of infection. By contrast, if the partner of individual $i$ is expected to undergo testing and if $i$ believes that his partner's test results will be observable, the propensity to test at $t_{1}, \Theta_{A-\Omega}$, is below the relative cost of testing $\frac{C}{H}$ if $P_{n}$ is greater than 0.56 . Thus, if the transmission rate $\beta$ is overestimated and if $i$ and $j$ have already engaged in risky sex, $i$ will be opportunistic and will rely on the fact that his partner is expected to undergo HIV testing. In this case, he will test at $t_{2}$ if he observes that $j$ is HIV-positive because $\Theta_{\Omega}$ is higher than the relative cost of testing. In this case, it is possible to encourage $i$ to undergo HIV testing by reducing $P_{n}$ through various methods, such as the promotion of home-based self-testing for HIV.

The promotion of testing through a reduction in $P_{n}$ is not possible if the cost of testing is high. In figure 2(b), the propensity $\Theta_{A-\Omega}$ is always lower than $\frac{C}{H}$; therefore, individuals will always behave opportunistically if they believe that their partner will undergo HIV testing and that the test results will be observable. Moreover, in this situation, the strategy of reducing $P_{n}$ may hamper individuals' willingness to be tested for HIV if these individuals believe that their partners will not undergo HIV testing or that their partners' test results will not be observable.

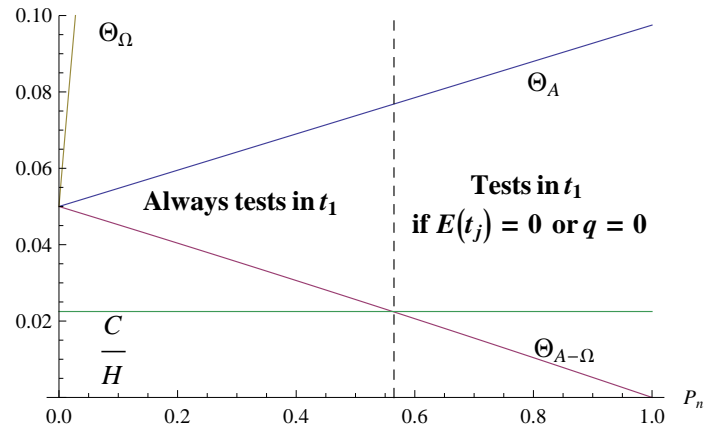

(a) If the relative testing price is low.

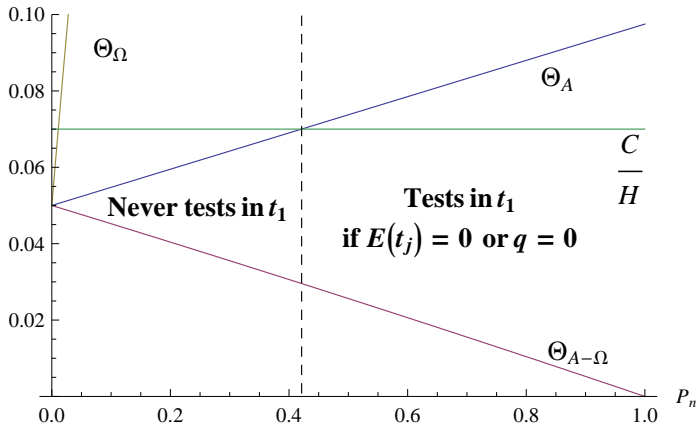

(b) If the relative testing price is high.

Figure 2: The propensities $\Theta_{A}, \Theta_{A-\Omega}$ and $\Theta_{\Omega}$ as a function of $P_{n}$.

Figures 3(a) and 4(a) also represent the propensities $\Theta_{A}$ and $\Theta_{A-\Omega}$; however, in these figures, $P_{n}$ is replaced with its functional form $P_{n}=\left[1-(1-\beta)^{n}\right]$, and $p_{i i}$ is a strictly increasing and concave function of $\beta$. The relative testing $\operatorname{cost} C / H$ is omitted to avoid overcomplicating these figures $(C / H$ would be represented by a horizontal plane). We consider and compare two scenarios for $p_{i i}$, that is, for the sexual background of $i$ prior to his relationship with $j$.

The first of these two scenarios assumes that $i$ had only one regular sexual partner, $k$, prior to his relationship with $j$. It is assumed that $i$ has had 100 unprotected encounters with $k$, and that the expected probability that $k$ was infected prior to the relationship between $i$ and $k$ is $p_{i k}=5 \%$. With these assumptions, $p_{i i}$ is equal to $p_{i i}=0.05\left[1-(1-\beta)^{100}\right]$. If the transmission rate is overestimated $(\beta \approx 1)$ and $n \geq 1$, then $p_{i i}$ is approximately equal to $5 \%$.

Figure 3(a) displays the propensities $\Theta_{A}$ and $\Theta_{A-\Omega}$ under this first scenario ${ }^{12}$. As shown in corollary 2.2, the propensity $\Theta_{A}$ is increasing in $n$ and $\beta$. By contrast, $\Theta_{A-\Omega}$ is an inverted U-shaped function of $\beta$ and is decreasing in $n$, as predicted by corollary 2.4. Figure 3(a) shows that $\Theta_{A-\Omega}$ is close to zero if the transmission rate is overestimated and if $i$ and $j$ have already engaged in unprotected sex. This implies that individuals will be opportunistic and postpone testing if they believe that their partners will be tested for HIV at $t_{1}$ and that the test results will be observable. In this situation, reductions in $n$ and $\beta$ are expected to increase the propensity $\Theta_{A-\Omega}$. If the testing cost is low, this strategy

\footnotetext{
${ }^{12}$ For clarity, the lower bound of $n$ is set equal to 0.2 in figures 3(a) and 4(a). If $n$ is equal to zero, $\Theta_{A}$ and $\Theta_{A-\Omega}$ are flat and coincidental.
} 
may be effective at increasing testing rates. Figure 3(b) indicates the area in which the condition $\Theta_{A-\Omega} \geq \frac{C}{H}$ is satisfied if the testing cost is low $(C=0.0225$, as in figure 2(a)). For example, with these assumptions, if $i$ and $j$ have already engaged in unprotected sex on five occasions $(n=5)$, individuals will be tempted to be opportunistic if $\beta$ is close to 1. By contrast, individuals will undergo HIV testing at $t_{1}$ if $\beta$ ranges between $0.6 \%$ and $15.2 \%$. If the testing cost increases, then this area is reduced, and vanishes altogether if $C \geq 0.05$.

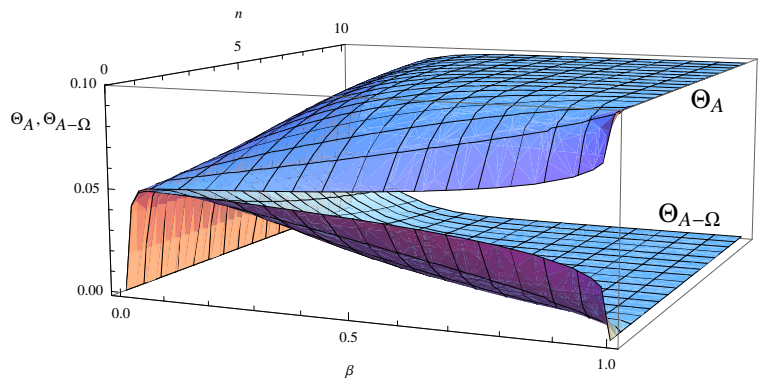

(a) The propensities $\Theta_{A}$ and $\Theta_{A-\Omega}$.

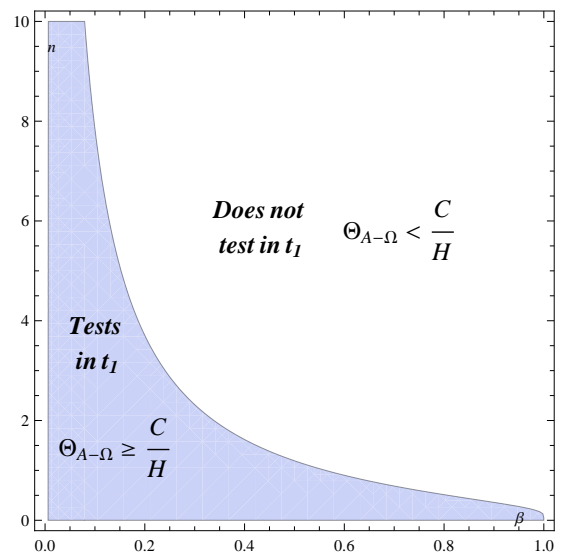

(b) The ranges of $n$ and $\beta$ in which $\Theta_{A-\Omega} \geq \frac{C}{H}$

Figure 3: First scenario: the propensities $\Theta_{A}$ and $\Theta_{A-\Omega}$ as a function of $\beta$ and $n$.

The second scenario assumes that $i$ has engaged in 10 casual encounters with different partners prior to his relationship with $j$. The expected probability that each of these partners was infected prior to engaging in a sexual relationship with $i$ is also equal to $5 \%$. Under these assumptions, $p_{i i}$ is equal to $p_{i i}=1-(1-0.05 \beta)^{10}$. The expected probability that $i$ is infected with HIV prior to his relationship with $j$ is much higher in this scenario than in the first scenario. If the transmission rate is overestimated $(\beta \approx 1)$ and $n \geq 1$, then $p_{i i}$ is approximately equal to $40 \%$.

Figure 4(a) displays the propensities $\Theta_{A}$ and $\Theta_{A-\Omega}$ under this second scenario. The positive relationship between $\Theta_{A}$ and $\beta$ still holds. In comparison with figure 3(a), the propensity $\Theta_{A}$ is higher because the expected probability $p_{i i}$ is larger under this second scenario. The propensity $\Theta_{A-\Omega}$ remains an inverted U-shaped function of $\beta$ and a decreasing function of $n$, as predicted by corollary 2.4. Figure 4(a) shows that $\Theta_{A-\Omega}$ is close to zero when $\beta$ and $n$ are high, implying that individuals will be opportunistic and postpone testing if they believe that their partners will be tested for $\mathrm{HIV}$ at $t_{1}$ and if the results of these tests are expected to be observable. In this case, reductions in $n$ and $\beta$ are expected to increase the propensity $\Theta_{A-\Omega}$. Similarly to the first scenario, if the testing cost is low, then this strategy is effective at increasing HIV testing rates. Figure 4(b) shows the area in which the condition $\Theta_{A-\Omega} \geq \frac{C}{H}$ is satisfied if the testing cost is low $(C=0.0225)$. For example, if $i$ and $j$ have already engaged in five occurrences of unprotected sex $(n=5)$, partners will be opportunistic if $\beta$ is close to 1 but will always choose to be tested for HIV at $t_{1}$ if $\beta$ is between $6.2 \%$ and $34.3 \%$. If the testing cost increases, this area is reduced; the area in question vanishes completely if $C \geq 0.40$.

\section{Strategic behavior: the observability of symptoms}

In the above discussion, the observability was defined to be the expected probability to observe the test results of a sexual partner. It was assumed that $H$, the expected benefit from HIV-related health care for infected individuals, was as high at $t_{1}$ as at $t_{2}$. This section considers another type of observability, namely, the observability of HIV/AIDS symptoms. It shows that a crucial parameter in the testing decision-making is individuals' beliefs about whether HIV treatment is required prior to the appearance of symptoms.

On the one hand, if treatment is thought to be less effective after the onset of AIDS symptoms, individuals who are at risk of infection are expected to undergo frequent HIV testing if they repeatedly engage in risky behaviors. On the 


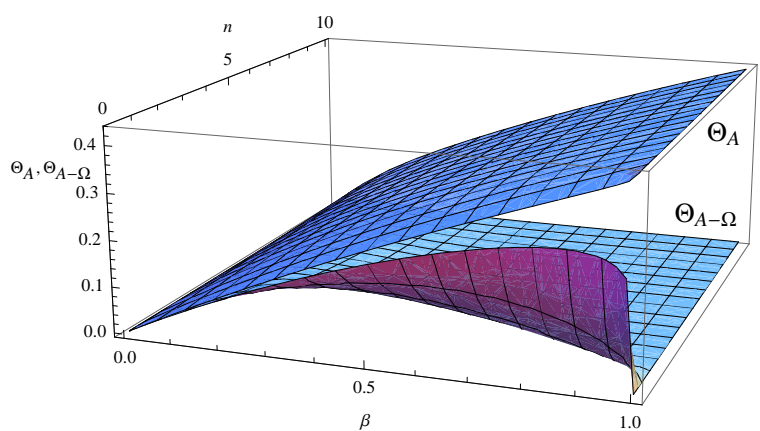

(a) The propensities $\Theta_{A}$ and $\Theta_{A-\Omega}$

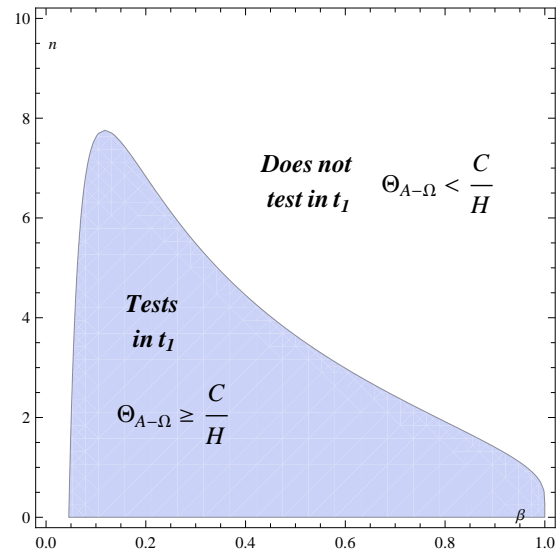

(b) The ranges of $n$ and $\beta$ in which $\Theta_{A-\Omega} \geq \frac{C}{H}$

Figure 4: Second scenario: the propensities $\Theta_{A}$ and $\Theta_{A-\Omega}$ as a function of $\beta$ and $n$.

other hand, if antiretroviral therapy is thought to be required only if HIV/AIDS is symptomatic, most individuals will postpone testing until they feel symptoms or observe that their partner is sick. These beliefs about the observability of HIV/AIDS symptoms sharply reduce the incentive to test and provide a possible explanation for the low rates of testing that have been observed in sub-Saharan Africa.

The existing literature confirms that late testing is common and that many persons who are at high risk of infection only undergo tests for HIV after the onset of AIDS symptoms (Creek et al., 2006; Bwambale et al., 2008; Wanyenze et al., 2011). The high rates of late testing are particularly detrimental because the early diagnosis of HIV infection has been shown to improve HIV treatment, decrease the cost of HIV-related medical care and reduce the infectivity of HIV (Girardi et al., 2007). Let us extend the framework of section 2 to address the possibility of late testing.

\subsection{The construction of the model}

The modified sequence of the decision-making process is depicted in figure 5 . At the time of the decision, $t_{1}$, the individual $i$ has already engaged in $n \geq 0$ risky encounters with his partner $j$. This individual must choose whether undergo an HIV test at $t_{1}$ and/or at $t_{2}$. To simplify the model, it is assumed that test results are not observable. If $i$ decides to test at $t_{1}$, he bears the testing cost $C$ but has access to HIV treatment if he is infected. The expected value of HIV treatment if the disease is asymptomatic is equal to $H_{1}$. After the decision at $t_{1}, i$ engages in $N \geq 0$ risky encounters with $j$.

The individual $i$ may also test at $t_{2}$, which would occur after the onset of symptoms if $i$ is infected. In this situation, $i$ also bears the testing $\operatorname{cost} C$. The expected value of HIV treatment after the onset of AIDS symptoms is equal to $H_{2}$. It is assumed that $H_{1} \geq H_{2}>C$ : if $i$ knows that he is infected or if AIDS symptoms appear, $i$ will undergo testing for HIV to obtain access to health care.

\subsection{The model solution}

Within this framework, the decision to test is strategic and largely depends on the difference $H_{1}-H_{2}$ between the expected values of health care before and after the onset of symptoms. If this difference is high, then at-risk individuals are expected to undergo HIV testing to benefit from better health care if the access to HIV treatment is widespread. By contrast, if $H_{1} \approx H_{2}$, HIV tests are only performed in symptomatic cases of AIDS. This reasoning is summarized in the following proposition.

Proposition 3.1. An individual $i$ will undergo testing for HIV before the onset of AIDS symptoms if:

$$
\omega=\underbrace{\left[p_{i i}+p_{i j}\left(1-p_{i i}\right) P_{n}\right]}_{\text {i infected at } t_{1}}\left(H_{1}-H_{2}\right)-\underbrace{\left(1-p_{i i}\right)\left(1-p_{i j} P_{n}\right)}_{\text {i not infected at } t_{1}} C \geq 0 .
$$




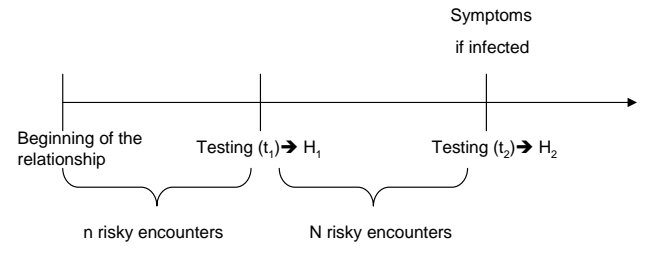

Figure 5: The sequence of the model.

This condition is satisfied if the expected probability that $i$ is infected at the time of the decision is high and if $H_{1}$, the expected value of health care if HIV/AIDS is asymptomatic, is significantly higher than $\mathrm{H}_{2}$, the expected value of health care after the onset of AIDS symptoms.

Proof The proof is in the appendix.

$\omega$ is the difference between the better quality of health care that $i$ receives if HIV is detected at $t_{1}$ and $i$ 's deadweight testing cost if he is not infected but undergoes HIV testing at $t_{1}$. It is notable that $\omega$ becomes larger as the expected probabilities $p_{i}, p_{j}$ and $P_{n}$ increase. This is unsurprising because a higher probability of HIV infection for $i$ at $t_{1}$ is associated with a higher expected cost from postponing HIV testing. As a consequence, $\omega$ is also increasing in $\beta$, the expected transmission rate.

Proposition 3.1 implies that individuals will only test after the onset of symptoms if they incorrectly believe that HIV treatment is only required for situations involving symptomatic cases of AIDS. However, in reality, the early diagnosis of HIV has been shown to improve treatment, reduce the cost of HIV-related medical care and reduce the infectivity of HIV (Girardi et al., 2007). Thus, if prevention campaigns succeed in correcting this misconception, atrisk persons may decide to be tested for HIV on a regular basis, particularly if the cost of testing is low and if these individuals frequently engage in risky behaviors.

In section 2 and 3, two mechanisms that reduce the willingness to be tested for HIV were presented. Both of these mechanisms are based on erroneous belief with respect to the HIV/AIDS virus. Of course, these misconceptions are not expected to be equally common among all individuals. Therefore, the next section examines how the predictions of the model relate to evidence from the existing literature.

\section{Testing patterns and empirical facts}

The theoretical models that were developed in the two previous sections demonstrated the ways in which misconceptions about HIV/AIDS may generate misleading strategic behavior with respect to testing and thereby reduce individuals' incentives to be tested for HIV. This section strives to summarize these findings and to discuss these results in the context of HIV in Africa. More specifically, it provides a review of relevant literature and reveals that the predictions of the model are consistent with qualitative and quantitative evidence from sub-Saharan Africa.

The existing literature advances three main reasons for HIV testing (Gersovitz, 2011). First, testing may be a signal that is requested by sexual partners prior to engaging in unprotected encounters with high-risk individuals (Philipson and Posner, 1993, 1995; Boozer and Philipson, 2000). Second, altruistic individuals who are concerned about the health of their partners may be willing to undergo HIV testing and to engage in safe sexual practices if they are infected (Philipson and Posner, 1993). As explained in the introduction of this paper, these two reasons for testing are inconsistent with the low testing rates that have been observed in sub-Saharan Africa. The high prevalence of HIV in this region should indeed encourage individuals to require HIV testing from their partners; moreover, altruistic 
individuals should be willing to undergo testing to protect their sexual partners. A third strand of the literature stresses the importance of the objective and subjective costs that are associated with testing and treatment (Gersovitz, 2011). It has been demonstrated that recent increases in access to testing and treatment and reductions in the cost of these processes are factors that increase individuals' willingness to be tested for HIV (Kaler and Watkins, 2010; Kautz, 2008; Thornton, 2008; Thielman et al., 2006; Wolff et al., 2005). Social stigma and fears are also frequently emphasized in the literature as factors that reduce HIV testing in sub-Saharan Africa (Obermeyer and Osborn, 2007; Bwambale et al., 2008; Kaler and Watkins, 2010). The analysis of this paper complements this literature by proposing two mechanisms that explain the low willingness of individuals to undergo HIV testing in sub-Saharan Africa.

The first mechanism that is developed in section 2 relates to the observation that individuals largely overestimate the transmission rate of HIV. Although the true per-act transmission rate of HIV is generally lower than $1 \%$, individuals' average estimates for the per-act transmission rate for HIV have been measured at above $80 \%$ in both Burundi and rural Malawi (Boily et al., 2009; Delavande and Kohler, 2009; Sterck, 2012a).

As addressed in the model that is constructed in this study, the overestimation of the infectivity of HIV implies that partners do not believe that serodiscordance is possible in committed sexual relationships. This misleading belief is consistently reported in the recent literature. The results from a survey that was conducted in Uganda indicate that $61.8 \%$ of the surveyed men do not believe that discordant HIV results could exist between two partners in a committed relationship (Bwambale et al., 2008). The qualitative literature includes numerous explanations from interviewees that reveal a belief that serodiscordance is impossible (Mlay et al., 2008; Bwambale et al., 2008; Bunnell et al., 2005; Santow et al., 2008; Ngure et al., 2012). For example, in a study by Santow et al. (2008), this belief is clearly evident in the following quote from a Malawian man:

"If my wife has the disease then it means I have it as well".

Similarly, Bwambale et al. (2008) presents the following thought from a Ugandan male:

"Some men have lost their wives due to HIV/AIDS but they lie to us that they were found HIV negative when they tested. This is not true”.

Santow et al. (2008) suggest that the disbelief in serodiscordance is reinforced by the analogizing of HIV to other sexually transmitted diseases, which are typically characterized by higher transmission rates. Misconceptions about serodiscordance are widespread among not only VTC clients but also counselors (Bunnell et al., 2005). These misconceptions include, among other explanations, the concept of hidden infections that are not detectable by HIV tests, a belief in immunity to HIV/AIDS and a belief in protection from God with respect to HIV/AIDS (Bunnell et al., 2005; Santow et al., 2008). Only a minority of respondents explain serodiscordance through the use of scientifically established factors (Bunnell et al., 2005).

Because partners do not believe in serodiscordance, only one partner from each committed couple is typically expected to undergo HIV testing, as the information that is provided by the results of an HIV test for one partner is expected to reflect the serostatus of both partners. Section 2 demonstrated that this type of strategic behavior is amplified by the overestimation of the transmission rate in the situation of individual decision-making regarding HIV testing. A similar conclusion has also been obtained for scenarios involving a joint decision with respect to HIV testing (Appendix B).

The literature confirms that denial of serodiscordance within a couple leads to low testing rates. After individuals have tested positive for HIV, their partners rarely seek HIV testing (Temmerman et al., 1995; Shetty et al., 2005). Gersovitz (2011) suggests that this phenomenon could be reduced if antiretroviral therapy was widely available and if individuals were better informed about the prevalence of discordant couples. The qualitative literature also indicates that strategic behaviors are frequent, but the importance of this phenomenon has not yet been quantified. This is well illustrated in the following conversation between two men in rural Malawi: 
"Yes, indeed, people say that lying together is dying together. If he has HIV/AIDS, I have HIV/AIDS, but I know that we don't have it." And I asked, "How do you know? Did you go for a blood test?" She said, "I know my own behavior, and he told me one day that he doesn't have HIV/AIDS. He went for a blood test and found that he doesn't have it"(Santow et al., 2008).

Similarly, King et al. (2008) reveals that the low levels of understanding on serodiscordance appears to encourage these HIV-positive individuals to more frequently disclose their serostatus to their partner and recommend HIV testing to their partner, as many HIV-positive individuals believe that their untested partners are also positive:

"When you tell your partner your HIV status, she...also goes for an HIV test and knows that she is also HIV-positive, she begins going for medical care.”.

The fact that people are tempted to infer their serostatus from misleading information may not only reduce testing rates but also produce harmful consequences with respect to condom use:

"Some partners who had not been tested assumed that they were HIV-positive. Increasing condom use with these partners was reportedly challenging”(Lifshay et al., 2009).

The model of section 2 is also consistent with the observations deduced from table 1. More specifically, the described mechanism provides a new explanation for the low testing rates in sub-Saharan Africa. This explanation is also consistent with the fact that frequently, only one of the members of sub-Saharan African couples undergoes testing for HIV. The measurement of the importance of this phenomenon is extremely important because over two-thirds of couples in sub-Saharan Africa with at least one partner who is infected are serodiscordant (de Walque, 2006). The annual incidence of HIV for the uninfected member of discordant couples is between 10 and 20\%; this incidence is between 10- and 100-fold higher than the incidence of HIV among corresponding individuals who are in (concordant) HIV-negative couples (Bunnell et al., 2005). This phenomenon implies that most HIV transmissions in sub-Saharan Africa occur within the context of marriage (Dunkle et al., 2008). The mechanism that is proposed in section 2 is therefore an important step for understanding the denial of serodiscordance and the impact of this denial on testing behavior.

The second mechanism that is presented in section 3 analyzes how decisions with respect to HIV testing are affected by expectations of how the benefits of HIV treatment evolve with the stages of HIV infection. The constructed model of this study indicates that individuals are expected to postpone HIV testing if ARV therapies are thought to only be required if the disease is symptomatic.

This mechanism is consistent with the high rates of late testing that are reported in the literature. From a survey that was conducted in a hospital in urban Uganda, Wanyenze et al. (2011) finds that 31.3\% of survey respondents were infected with HIV, and CD4 counts that were less than 250 cells $/ \mathrm{mm}^{3}$ were found in $47.6 \%$ of these infected individuals ${ }^{13}$. In this study, $46.2 \%$ of testers reported concerns about illness/AIDS symptoms. Similarly, Creek et al. (2006) find that $20.1 \%$ of VTC clients in Botswana sought a test for symptom-related reasons, and $77.7 \%$ of these individuals were HIV-positive. In another study in Uganda, $15.3 \%$ of men reported frequent illnesses as their reason for seeking HIV testing (Bwambale et al., 2008).

The model of section 3 shows that individuals will test late if the effectiveness of HIV treatment is thought to be required only if HIV/AIDS is symptomatic. This misconception is highlighted in the qualitative literature. For example, Kaler and Watkins (2010) report the following conversation between two men in a bus in Malawi:

One said he was going to Zomba for voluntary counseling and testing. Then the other man asked him, while puzzled, saying "[why] have one's blood tested, why [not] just staying [without testing]? If one has got HIV/AIDS he will start to get sick, [there is no need] to go and have one's blood tested".

\footnotetext{
${ }^{13} \mathrm{An}$ advanced HIV infection corresponds to a CD4 count that is at or below 350 cells $/ \mathrm{mm}^{3}$ (in accordance with WHO guidelines that were enacted prior to 2009).
} 
Even more serious is the frequently held belief that learning about an HIV-positive status will be detrimental to an individual's health (Obermeyer and Osborn, 2007). The qualitative literature documents several instances of people who are unwilling to be tested for HIV out of fear that the disease would become worse. This common fear is well summarized by the following quotes:

"It is better for her to live ignorant, without knowing the truth for she will live happy without worrying about anything” (Kaler and Watkins, 2010).

"You can die very soon if you tested positive because of worries" (Bwambale et al., 2008).

In accordance with the notation of section 3, this evidence suggests that individuals frequently believe that $H_{1}<$ $H_{2}$. In this situation, $\omega$ is negative, and individuals always prefer to be tested at later times, after the onset of AIDS symptoms (equation (8)).

Misconceptions about HIV treatment and HIV stages provide a realistic explanation for the high rates of late testing in sub-Saharan Africa. This phenomenon is particularly concerning because the early diagnosis of HIV infection has been shown to be critical for maximizing the effectiveness of HIV treatment, decreasing the cost of HIV-related medical care and reducing the infectivity of HIV (Girardi et al., 2007). Furthermore, early testing may reduce risky behavior if people are altruistic and believe that serodiscordance is possible (Sterck, 2012a).

\section{Conclusion}

Voluntary testing and counseling (VTC) is considered to be one of the most powerful policy tools that is available for fighting the HIV/AIDS epidemic. Early testing and treatment for HIV are associated with improved health outcomes and reduced infectivity. Furthermore, testing may foster safer behavior among tested individuals who are either HIV-positive and altruistic or HIV-negative and fearful. Despite this, testing services are underused in sub-Saharan Africa, and it is rare for both members of a couple to be tested for HIV.

The aim of this paper was to explain these empirical puzzles. It complements the existing literature by examining how misconceptions about HIV infectivity and HIV treatments encourage strategic behavior and thereby explain the low rates of HIV testing in sub-Saharan Africa. Two mechanisms were emphasized.

The first theoretical framework focused on strategic interactions that relate to the observation of a partner's serostatus. It showed that individuals who are at risk for HIV may postpone testing if they believe that their partner will be tested for HIV and that these test results will be observable. The analysis showed that this strategic behavior is likely to occur because people generally overestimate the transmission rate of HIV and therefore do not believe that serodiscordance is possible within the context of a committed, sexually active couple.

The second theoretical framework examined how the decision to undergo HIV testing is affected by beliefs about the effectiveness of HIV treatment in various stages of HIV/AIDS. In general, the model predicts late testing if HIV therapies are thought to be needed only if HIV/AIDS is symptomatic.

The two aforementioned mechanisms are primarily caused by misconceptions. These mechanisms explain the low testing rates in sub-Saharan Africa and the high proportion of couples in this region that choose to have only one member of the couple tested for HIV. These mechanisms also explain the high rate of individuals who undergo HIV testing only after reaching an advanced stage of AIDS. The review of the literature supported the existence of these two phenomena. However, specific data are needed to measure the importance of the mechanisms.

Let us conclude this paper by discussing the policy implications that may be derived from the analysis of this study. First, an unambiguous policy to enhance HIV testing rates would involve increasing access to HIV treatment and reducing not only the direct cost of HIV testing but also its indirect costs which relate to social stigmas and fears. Second, the dissemination of the fact that the transmission rate of HIV is lower than many individuals predict may reduce the general belief that serodiscordance is impossible in regular relationships and thereby reduce the propensity 
to act strategically by postponing testing. However, this prediction should be regarded with caution because the disclosure that HIV has a transmission rate that is lower than expected may foster riskier behavior. Third, our analysis suggests that the promotion of home-based self-testing may sharply increase testing; this approach would not only increase access to HIV testing and reduce testing costs but also minimize strategic behavior by reducing the number of unprotected encounters that preceded a decision to undergo HIV testing. This policy is extremely relevant in the context of sub-Saharan Africa because the high prevalence of concurrent long-term relationships in this region implies a need to be regularly tested for HIV (Halperin and Epstein, 2004; Mah and Halperin, 2010). Finally, this paper highlights the importance of prevention campaigns that combat common misconceptions about HIV/AIDS; in particular, these campaigns should address misconceptions about serodiscordance within couples and the efficacy of HIV treatments at various stages of HIV/AIDS.

Boily, M., R. Baggaley, L. Wang, B. Masse, R. White, R. Hayes, M. Alary, et al. (2009). Heterosexual risk of HIV-1 infection per sexual act: systematic review and meta-analysis of observational studies. The Lancet infectious diseases 9(2), 118-129.

Boozer, M. and T. Philipson (2000). The impact of public testing for human immunodeficiency virus. Journal of Human Resources 35(3), 419-446.

Bunnell, R., J. Nassozi, E. Marum, J. Mubangizi, S. Malamba, B. Dillon, J. Kalule, J. Bahizi, N. Musoke, and J. Mermin (2005). Living with discordance: knowledge, challenges, and prevention strategies of HIV-discordant couples in Uganda. AIDS care 17(8), 999-1012.

Bwambale, F., S. Ssali, S. Byaruhanga, J. Kalyango, and C. Karamagi (2008). Voluntary HIV counselling and testing among men in rural Western Uganda: Implications for prevention. BMC Public Health 8(1), 1-12.

Castilla, J., J. D. Romero, V. Hernando, B. Marincovich, S. García, and C. Rodríguez (2005). Effectiveness of highly active antiretroviral therapy in reducing heterosexual transmission of HIV. Journal of Acquired Immune Deficiency Syndromes (1999) 40(1), 96-101.

Chimbiri, A. M. (2007). The condom is an 'intruder' in marriage: Evidence from rural Malawi. Social Science $\mathcal{E}$ Medicine 64(5), $1102-1115$.

Creek, T., M. Alwano, R. Molosiwa, T. Roels, T. Kenyon, V. Mwasalla, E. Lloyd, M. Mokomane, P. Hastings, A. Taylor, et al. (2006). Botswana's tebelopele voluntary HIV counseling and testing network: use and client risk factors for HIV infection, 2000-2004. JAIDS Journal of Acquired Immune Deficiency Syndromes 43(2), 210-218.

de Walque, D. (2006). Who gets AIDS and how? The determinants of HIV infection and sexual behaviors in Burkina Faso, Cameroon, Ghana, Kenya, and Tanzania. World Bank Policy Research Working Paper No. 3844.

Delavande, A. and H. Kohler (2009). The impact of HIV testing on subjective expectations and risky behavior in Malawi. Demography, 1-26.

Dunkle, K., R. Stephenson, E. Karita, E. Chomba, K. Kayitenkore, C. Vwalika, L. Greenberg, S. Allen, et al. (2008). New heterosexually transmitted HIV infections in married or cohabiting couples in urban Zambia and Rwanda: an analysis of survey and clinical data. Lancet 371(9631), $2183-2191$.

Flood, M. (2003). Lust, trust and latex: Why young heterosexual men do not use condoms. Culture, Health $\mathcal{E}$ Sexuality 5(4), 353-369.

Gersovitz, M. (2011). HIV testing: Principles and practice. The World Bank Research Observer 26(1), 1-41.

Gersovitz, M. and J. Hammer (2003). Infectious diseases, public policy, and the marriage of economics and epidemiology. The world bank research observer 18(2), 129-157.

Girardi, E., C. Sabin, and M. Antonella d'Arminio Monforte (2007). Late diagnosis of HIV infection: epidemiological features, consequences and strategies to encourage earlier testing. JAIDS Journal of Acquired Immune Deficiency Syndromes 46, S3-S8.

Gong, E. (2010). HIV Testing \& Risky Sexual Behavior. Mimeo.

Halperin, D. and H. Epstein (2004). Concurrent sexual partnerships help to explain Africa's high HIV prevalence: implications for prevention. The Lancet 364(9428), 4-6.

Kaler, A. and S. Watkins (2010). Asking god about the date you will die: HIV testing as a zone of uncertainty in rural Malawi. Demographic research 23(32), 905.

Kaplan, E. H. (1990). Modeling HIV infectivity: must sex acts be counted? Journal of Acquired Immune Deficiency Syndromes 3(1), 55-61.

Kautz, T. (2008). Stigma, fear and hope: A model of HIV testing in Sub-Saharan Africa. Mimeo.

King, R., D. Katuntu, J. Lifshay, L. Packel, R. Batamwita, S. Nakayiwa, B. Abang, F. Babirye, P. Lindkvist, E. Johansson, et al. (2008). Processes and outcomes of HIV serostatus disclosure to sexual partners among people living with HIV in Uganda. AIDS and Behavior 12(2), 232-243.

Knauper, B. and R. Kornik (2004). Perceived transmissibility of stis: lack of differentiation between HIV and chlamydia. Sexually transmitted infections $80(1), 74$.

Lifshay, J., S. Nakayiwa, R. King, O. Reznick, D. Katuntu, R. Batamwita, E. Ezati, A. Coutinho, C. Kazibwe, and R. Bunnell (2009). Partners at risk: motivations, strategies, and challenges to HIV transmission risk reduction among HIV-infected men and women in Uganda. AIDS care 21(6), 715-724.

Linville, P., G. Fischer, and B. Fischhoff (1993). AIDS risk perceptions and decision biases.

Mah, T. and D. Halperin (2010). Concurrent sexual partnerships and the HIV epidemics in Africa: evidence to move forward. AIDS and Behavior 14(1), 11-16.

Maticka-Tyndale, E. and C. Kyeremeh (2010). The trouble with condoms: norms and meanings of sexuality and condom use among school-going youth in Kenya. International Journal of Sexual Health 22(4), 234-247.

Medley, A., C. Garcia-Moreno, S. McGill, and S. Maman (2004). Rates, barriers and outcomes of HIV serostatus disclosure among women in developing countries: implications for prevention of mother-to-child transmission programmes. Bulletin of the World Health Organization 82, 299-307.

Meekers, D., M. Klein, and L. Foyet (2003). Patterns of HIV risk behavior and condom use among youth in Yaoundé and Douala, Cameroon. AIDS and Behavior 7(4), 413-420.

Mlay, R., H. Lugina, and S. Becker (2008). Couple counselling and testing for HIV at antenatal clinics: views from men, women and counsellors. AIDS care 20(3), 356-360.

Moore, A. and J. Oppong (2007). Sexual risk behavior among people living with HIV/AIDS in togo. Social science E medicine 64(5), $1057-1066$. 
Ngure, K., N. Mugo, C. Celum, J. Baeten, M. Morris, O. Olungah, J. Olenja, H. Tamooh, and B. Shell-Duncan (2012). A qualitative study of barriers to consistent condom use among HIV-1 serodiscordant couples in Kenya. AIDS care 24(4), 509-516.

Obermeyer, C. and M. Osborn (2007). The utilization of testing and counseling for HIV: a review of the social and behavioral evidence. American Journal of Public Health 97(10), 1762.

O’Donoghue, T. and M. Rabin (2001). Risky behavior among youths: Some issues from behavioral economics. In Risky Behavior among Youths: An Economic Analysis, NBER Chapters, pp. 29-68. National Bureau of Economic Research, Inc.

Philipson, T. and R. Posner (1993). Private choices and public health: The AIDS epidemic in an economic perspective. Harvard University Press.

Philipson, T. and R. Posner (1995). A theoretical and empirical investigation of the effects of public health subsidies for std testing. The Quarterly Journal of Economics 110(2), 445-474.

Pinkerton, S., L. Wagner-Raphael, C. Craun, and P. Abramson (2000). A quantitative study of the accuracy of college students' HIV risk estimates1. Journal of Applied Biobehavioral Research 5(1), 1-25.

Rhodes, T. (1997). Risk theory in epidemic times: sex, drugs and the social organisation of 'risk behaviour'. Sociology of Health $\mathcal{E}$ Illness 19(2), $208-227$.

Rottingen, J. and G. P. Garnett (2002). The epidemiological and control implications of HIV transmission probabilities within partnerships. Sexually Transmitted Diseases 29(12), 818-827.

Sanders, S., W. Yarber, E. Kaufman, R. Crosby, C. Graham, and R. Milhausen (2012). Condom use errors and problems: a global view. Sexual Health 9(1), 81-95.

Santow, G., M. Bracher, and S. Watkins (2008). Implications for behavioural change in rural Malawi of popular understandings of the epidemiology of AIDS. In Paper presented at IUSSP Seminar on Potential and Actual Contributions of Behavioral Change to Curbing the Spread of HIV. Entebbe, Uganda.

Shetty, A., M. Mhazo, S. Moyo, A. von Lieven, P. Mateta, D. Katzenstein, Y. Maldonado, D. Hill, and M. Bassett (2005). The feasibility of voluntary counselling and HIV testing for pregnant women using community volunteers in Zimbabwe. International journal of STD $\mathcal{E}$ AIDS 16(11), 755-759.

Sterck, O. (2012a). HIV/AIDS and fatalism: Should prevention campaigns disclose the transmission rate of HIV? Mimeo.

Sterck, O. (2012b). Why only one of the partners tests for HIV/AIDS among sub-Saharan African couples? SSRN working paper.

Temmerman, M., J. Ndinya-Achola, J. Ambani, P. Piot, et al. (1995). The right not to know HIV-test results. Lancet 345(8955), 969.

Thielman, N., H. Chu, J. Ostermann, D. Itemba, A. Mgonja, S. Mtweve, J. Bartlett, J. Shao, and J. Crump (2006). Cost-effectiveness of free HIV voluntary counseling and testing through a community-based AIDS service organization in Northern Tanzania. Journal Information 96(1).

Thornton, R. L. (2008). The demand for, and impact of, learning HIV status. American Economic Review 98(5), $1829-1863$.

Wanyenze, R., M. Kamya, R. Fatch, H. Mayanja-Kizza, S. Baveewo, S. Sawires, D. Bangsberg, T. Coates, and J. Hahn (2011). Missed opportunities for HIV testing and late-stage diagnosis among HIV-infected patients in Uganda. PLoS One 6(7), 1-11.

Wolff, B., B. Nyanzi, G. Katongole, D. Ssesanga, A. Ruberantwari, and J. Whitworth (2005). Evaluation of a home-based voluntary counselling and testing intervention in rural Uganda. Health Policy and Planning 20(2), 109-116.

Who, UnAIDs \& UnICEF (2009). Towards universal access: Scaling up priority HIV/AIDS interventions in the health sector. Progress report. Geneva.

Who, UnAIDs \& UnICEF (2010). Global report: UnAIDs report on the global AIDS epidemic 2010.

\section{Appendix A. Proofs}

Corollary 2.2. The propensity $\Theta_{A}$ is an increasing function of the expected transmission rate per act $\beta$. Therefore, if the test results of the individual's partner are expected to be unobservable, then the individual's overestimation of the HIV transmission rate may promote his decision to be tested for HIV.

Proof The derivative of $\Theta_{A}$ with respect to $\beta$ is given by:

$$
\frac{\partial \Theta_{A}}{\partial \beta}=p_{i i}^{\prime}\left(1-p_{i j} P_{n}\right)+\left(1-p_{i i}\right)\left(p_{i j}^{\prime} P_{n}+p_{i j} P_{n}^{\prime}\right)
$$

We conclude that $\frac{\partial \Theta_{A}}{\partial \beta}$ is strictly positive if $p_{i i}^{\prime}>0, p_{i j}^{\prime}>0$ and $P_{n}^{\prime}>0$.

Proposition 2.3. If $j$ is not expected to test or if $i$ and $j$ have never engaged in unprotected $\operatorname{sex}(n=0)$, then the individual $i$ tests for $H I V$ at $t_{1}$ if $A \geq 0$.

On the contrary, if $j$ is expected to test at $t_{1}$ and if $i$ and $j$ have had $n>0$ unprotected encounters before $t_{1}$, then the incentive for $i$ to be tested at $t_{1}$ is reduced by opportunism. In this case, i tests at $t_{1}$ if $A-\Omega \geq 0$, where the opportunism $\Omega$ is defined as follows:

$$
\Omega=\underbrace{\left[p_{i i} p_{i j}+p_{i i}\left(1-p_{i j}\right) P_{n}+\left(1-p_{i i}\right) p_{i j} P_{n}\right]}_{\text {both } \text { i and } j \text { are infected at } t_{1}} H-\underbrace{\left[p_{i j}+p_{i i}\left(1-p_{i j}\right) P_{n}\right]}_{j \text { is infected at } t_{1}} C .
$$


If $i$ does not undergo HIV testing at $t_{1}$, he will undergo HIV testing at $t_{2}$ if he observes that his partner is HIV-positive and if $\Omega \geq 0$.

Proof If $i$ believes that $j$ will not undergo HIV testing at $t_{1}$, then the situation is similar to proposition (2.1), and $i$ will therefore chooses to be tested for HIV if $A \geq 0$. Similarly, if $n=0$, then the test results for $j$ will not be informative to $i$, and $i$ will again choose to be tested for HIV if $A \geq 0$.

If $i$ thinks that $j$ will choose to be tested for HIV, then $i$ has three possible courses of action. First, he may be tested for HIV at $t_{1}$ because this testing would allow him to obtain access to HIV-related health care benefits if he is infected. In this situation, if he tests negative and observes that his partner is infected, recall the assumption that $i$ will choose to engage in $N$ unprotected encounters with a new partner whose expected probability of being infected is $p_{i j}$. Therefore, if $i$ tests at $t_{1}$, his expected lifetime utility may be stated as follows:

$$
V\left(t_{1}^{i}=1 \mid t_{1}^{j}=1\right)=U_{N}+\underbrace{\left[p_{i i}+p_{i j}\left(1-p_{i i}\right) P_{n}\right]}_{\text {i infected at } t_{1}}(H-d)-\underbrace{p_{i j}\left(1-p_{i i}\right)\left(1-P_{n}\right)}_{\begin{array}{c}
i \text { uninfected and } \\
j \text { infected }
\end{array}} \underbrace{p_{i j} P_{N} d}_{\begin{array}{c}
i \text { is infected by } \\
\text { his new partner }
\end{array}}-C .
$$

Second, $i$ may not undergo HIV testing at $t_{1}$ but may instead undergo this testing at $t_{2}$ if $j$ is observed to be HIV-positive. In this case, $i$ tests and benefits from health care only if both $i$ and $j$ are infected at the time of the testing decision. If $i$ tests negative at $t_{2}$, it is assumed that he finds a new partner for $N$ unprotected sexual encounters. Because $i$ does not undergo testing at $t_{1}$, if $i$ is infected but $j$ is uninfected at this time, $i$ is therefore excluded from receiving $H-C$, the net benefit of health care from being tested for HIV. His expected lifetime utility may then be stated as follows:

$$
\begin{aligned}
V\left(t_{1}^{i}=0, t_{2}^{i}=1 \mid t_{1}^{j}=1\right)= & U_{N}+\underbrace{\left[p_{i i} p_{i j}+p_{i i}\left(1-p_{i j}\right) P_{n}+\left(1-p_{i i}\right) p_{i j} P_{n}\right]}_{\text {both } i \text { and } j \text { infected at } t_{1}}(H-d-C)-\underbrace{p_{i i}\left(1-p_{i j}\right)\left(1-P_{n}\right)}_{\begin{array}{c}
i \text { infected and } \\
j \text { uninfected }
\end{array}} d \\
& -\underbrace{p_{i j}\left(1-p_{i i}\right)\left(1-P_{n}\right)}_{\begin{array}{c}
i \text { uninfected and } \\
j \text { infected }
\end{array}}(\underbrace{p_{i j} P_{N} d}_{\begin{array}{c}
i \text { is infected by } \\
\text { his new partner }
\end{array}}+C) .
\end{aligned}
$$

Finally, if the testing cost is high compared with its expected benefits, $i$ tests at neither $t_{1}$ nor $t_{2}$. In this case, he does not obtain any benefits from HIV-related health care if he is infected. Again, it is assumed that he finds a new sexual partner if he observes that $j$ is infected. His expected lifetime utility may then be expressed as follows:

$$
V\left(t_{1}^{i}=0, t_{2}^{i}=0 \mid t_{1}^{j}=1\right)=U_{N}-\underbrace{\left[p_{i i}+p_{i j}\left(1-p_{i i}\right) P_{n}\right]}_{\text {i infected at } t_{1}} d-\underbrace{p_{i j}\left(1-p_{i i}\right)\left(1-P_{n}\right)}_{\begin{array}{c}
i \text { uninfected and } \\
j \text { infected }
\end{array}} \underbrace{p_{i j} P_{N} d}_{\begin{array}{c}
i \text { is infected by } \\
\text { his new partner }
\end{array}} .
$$

Let us compare these three alternative courses of action. First, $i$ prefers to be tested for HIV at $t_{1}$ instead of $t_{2}$ if the following condition holds:

$$
\begin{aligned}
V\left(t_{1}^{i}=1 \mid t_{1}^{j}=1\right)-V\left(t_{1}^{i}=0, t_{2}^{i}=1 \mid t_{1}^{j}=1\right) & =\underbrace{p_{i i}\left(1-p_{i j}\right)\left(1-P_{n}\right)}_{\substack{i \text { infected and } \\
j \text { uninfected }}} H-\underbrace{\left[\left(1-p_{i i}\right)\left(1-p_{i j}\right)+p_{i i}\left(1-p_{i j}\right)\left(1-P_{n}\right)\right]}_{\text {j uninfected at } t_{1}} C \geq 0 \\
& \Leftrightarrow A-\Omega \geq 0,
\end{aligned}
$$

where opportunism $\Omega$ is defined as follows: $\Omega=\left[p_{i i} p_{i j}+p_{i i}\left(1-p_{i j}\right) P_{n}+\left(1-p_{i i}\right) p_{i j} P_{n}\right] H-\left[p_{i j}+p_{i i}\left(1-p_{i j}\right) P_{n}\right] C$. Secondly, if $i$ does not want to be tested for HIV at $t_{1}$ because $A-\Omega<0$, he nevertheless undergoes this testing at $t_{2}$ if $V\left(t_{1}^{i}=0, t_{2}^{i}=1 \mid t_{1}^{j}=1\right)-V\left(t_{1}^{i}=0, t_{2}^{i}=0 \mid t_{1}^{j}=1\right)=\Omega \geq 0$. Finally, if both $A-\Omega<0$ and $\Omega<0$ are satisfied, $i$ tests at neither $t_{1}$ nor $t_{2}$. 
Corollary 2.4. The propensity $\Theta_{A}$ is an increasing function of $\beta$, the expected per-act transmission rate. Therefore, if the HIV test results of an individual's partner are expected to be unobservable or if this partner is not expected to be tested for HIV, the overestimation of the transmission rate may foster testing.

By contrast, the propensity $\Theta_{A-\Omega}$ to test at $t_{1}$ is an inverted $U$-shaped function of $\beta$. As a consequence, if $j$ is expected to be tested for HIV at $t_{1}$ and if the test results are expected to be observable, the reduction of the expected transmission rate to an intermediate level $\beta^{*}$ would encourage $i$ to be tested at $t_{1}$ if there exists a $\beta^{*}<1$ such that $\Theta_{A-\Omega}\left(\beta^{*}\right) \geq 0$.

Proof The first part of this proof is similar to the proof of corollary 2.2.

To prove the second statement, let us demonstrate that $\Theta_{A-\Omega}$ is an inverted U-shaped function of $\beta$ if $p_{i i}^{\prime}>0$ and $P_{n}^{\prime}>0$ and $P_{n}(0)=p_{i i}(0)=0$. Note that $\Theta_{A-\Omega} \geq 0, \Theta_{A-\Omega}(\beta=0)=0$ and $\Theta_{A-\Omega}(\beta=1)=0$. Taking the derivative of $\Theta_{A-\Omega}$ with respect to $\beta$ produces the following expression:

$$
\frac{\partial \Theta_{A-\Omega}}{\partial \beta}=\frac{\left(1-P_{n}\right) p_{i i}^{\prime}-\left(1-p_{i i}\right) p_{i i} P_{n}^{\prime}}{\left[1-p_{i i} P_{n}\right]^{2}}
$$

The denominator of the above fraction is strictly positive. The numerator is continuous, positive for $\beta=0$, and negative for $\beta=1$. We conclude that $\Theta_{A-\Omega}$ is a U-shaped function of $\beta$ with an interior maximum between 0 and 1. Because $\Theta_{A-\Omega}(\beta=1)=0, i$ will never want to be tested for HIV if $\beta \approx 1, n>0$ and $E\left(t_{j}\right)=1$. In this case, if $\Theta_{A-\Omega}\left(\beta=\beta^{*}\right) \geq \frac{C}{H}$, a reduction of $\beta$ would encourage $i$ to test at $t_{1}$.

Proposition 3.1. An individual $i$ will undergo testing for HIV before the onset of AIDS symptoms if:

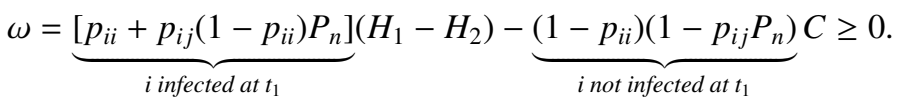

This condition is satisfied if the expected probability that $i$ is infected at the time of the decision is high and if $H_{1}$, the expected value of health care if HIV/AIDS is asymptomatic, is significantly higher than $\mathrm{H}_{2}$, the expected value of health care after the onset of AIDS symptoms.

Proof Because we assumed that $H_{2}>C$, the individual $i$ always undergoes HIV testing at $t_{2}$ if he has begun to experience AIDS symptoms. Two cases should therefore be compared. First, $i$ may be tested for HIV at $t_{1}$ and at $t_{2}$ if symptoms appear; alternatively, $i$ may only undergo testing at $t_{2}$ after the onset of AIDS symptoms. The lifetime expected utility of testing both at $t_{1}$ and at $t_{2}$ if AIDS symptoms appear is given by the following expression:

$$
V\left(t_{1}^{i}=1, t_{2}^{i}=1\right)=U_{N}-C+\underbrace{\left[p_{i i}+p_{i j}\left(1-p_{i i}\right) P_{n}\right]}_{i \text { infected at } t_{1}}\left(H_{1}-d\right)+\underbrace{p_{i j}\left(1-p_{i i}\right)\left(1-P_{n}\right) P_{N}}_{i \text { infected between } t_{1} \text { and } t_{2}}\left(H_{2}-C-d\right) .
$$

Similarly, the lifetime expected utility of testing at only $t_{2}$ is given by:

$$
V\left(t_{1}^{i}=0, t_{2}^{i}=1\right)=U_{N}+\underbrace{\left[p_{i i}+p_{i j}\left(1-p_{i i}\right) P_{n}+p_{i j}\left(1-p_{i i}\right)\left(1-P_{n}\right) P_{N}\right]}_{i \text { infected at } t_{2}}\left(H_{2}-C-d\right) .
$$

We define $\omega$ as the net benefit from testing at $t_{1}$ prior to the onset of symptoms, that is, as the difference between the two last equations:

$$
\omega \equiv V\left(t_{1}^{i}=1, t_{2}^{i}=1\right)-V\left(t_{1}^{i}=1, t_{2}^{i}=0\right)=\left[p_{i i}+p_{i j}\left(1-p_{i i}\right) P_{n}\right]\left(H_{1}-H_{2}\right)-\left(1-p_{i i}\right)\left(1-p_{i j} P_{n}\right) C .
$$

The individual $i$ will undergo testing for HIV before the emergence of symptoms if $\omega$ is positive. 


\section{Appendix B. Household model}

In section 2, it was assumed that each partner separately decides whether to be tested for HIV. This section reveals that the strategic mechanisms that are discussed in section 2 continue to hold if the hypothesis of individual decisionmaking is relaxed; in particular, this section examines the results if the decision to undergo HIV testing is reached in cooperation with a committed sexual partner and if the couple's utility function is maximized.

We begin by extending the notation of section 2 . In this section, the expected probability that $i$ is infected is denoted by $p_{i}$ and the expected probability that $j$ is infected is denoted by $p_{j}$. If only one of the partners tests positive, the other is assumed to have $N$ unprotected encounters with another individual, $k$, whose expected probability to be infected is denoted by $p_{k}$. We utilize $A_{i}$ and $A_{j}$ to denote the net benefit of testing at $t_{1}$ for $i$ and $j$ if their testing decisions are reached individually and if $q=0$ (the equivalent of $A$ in section 2). Similarly, $\Omega_{i}$ and $\Omega_{j}$ are defined to be the equivalents of $\Omega$, the opportunism metric that was introduced in section $2^{14}$.

If the decision to undergo HIV testing is reached in combination with a committed sexual partner, then proposition 2.3 is slightly modified by the presence of implicit altruism. The intuition underlying the presence of implicit altruism in the testing decision may be outlined as follows. Without loss of generality, let us assume that $j$ undergoes HIV testing at $t_{1}$. Her partner $i$ will benefit from this information in two ways. First, if $j$ is revealed to be HIV-positive, $i$ will learn that he is also likely to be infected with HIV (if $n>0$ ). In this case, he may test at $t_{2}$, leading to an expected gain of $\Omega_{i}$. Second, $i$ is provided with the opportunity to change partners and to thereby reduce his probability of becoming infected with HIV in the future. This phenomenon leads to an expected utility gain $E_{j}$ that is equal to the following expression:

$$
E_{j}=d \underbrace{p_{j}\left(1-p_{i}\right)\left(1-P_{n}\right)}_{\substack{i \text { uninfected and } \\
j \text { infected at } t_{1}}} \underbrace{\left(1-p_{k}\right) P_{N}}_{\begin{array}{c}
\text { Reduced risk with } \\
\text { a new partner } k
\end{array}} .
$$

$E_{j}$ should be regarded as a positive externality from $j$ to $i$; if $i$ is uninfected and if $j$ tests positive, then $i$ will change partners and reduce his probability of dying from AIDS. Similarly, the externality $E_{i}$ if $i$ tests for HIV may be defined as follows:

$$
E_{i}=d \underbrace{p_{i}\left(1-p_{j}\right)\left(1-P_{n}\right)}_{\begin{array}{c}
i \text { infected and } \\
j \text { uninfected at } t_{1}
\end{array}} \underbrace{\left(1-p_{k}\right) P_{N}}_{\begin{array}{c}
\text { Reduced risk with } \\
\text { a new partner } k
\end{array}} .
$$

In summary, if the decision to undergo HIV testing is jointly reached with a sexual partner, then testing is valued not only because it provides access to health care but also because it informs an individual's partner and protects him or her from the virus. This phenomenon is summarized in the following proposition, which is the equivalent of proposition 2.3 for a decision to be tested for HIV that is reached in combination with a sexual partner.

Proposition Appendix B.1. We assume that a decision to test for HIV is reached jointly with a committed sexual partner, that an individual's test results are disclosed to his or her partner $(q=1)$ and that $j$ is expected to be at greater risk for HIV infection than $i\left(p_{j}>p_{i}\right)$. The couple must to compare four alternatives. First, both partners will test for HIV at $t_{1}$ if opportunism is low and externalities are high, that is, if:

$$
\left\{\begin{array}{l}
A_{i}-\Omega_{i}+E_{i} \geq 0 \\
A_{i}+E_{i} \geq 0
\end{array}\right.
$$

These conditions are satisfied if $i$ and $j$ are likely to be infected and if the test results of the partners are not expected to be informative (if $P_{n}$ is low).

\footnotetext{
${ }^{14}$ Using this notation, $A_{i}=\left[p_{i}+p_{j}\left(1-p_{i}\right) P_{n}\right] H-C, A_{j}=\left[p_{j}+p_{i}\left(1-p_{j}\right) P_{n}\right] H-C, \Omega_{i}=\left[p_{i} p_{j}+p_{i}\left(1-p_{j}\right) P_{n}+\left(1-p_{i}\right) p_{j} P_{n}\right] H-\left[p_{j}+p_{i}\left(1-p_{j}\right) P_{n}\right] C$, and $\Omega_{j}=\left[p_{j} p_{i}+p_{j}\left(1-p_{i}\right) P_{n}+\left(1-p_{j}\right) p_{i} P_{n}\right] H-\left[p_{i}+p_{j}\left(1-p_{i}\right) P_{n}\right] C$.
} 
Second, only the partner who is at greater risk of HIV infection, $j$, will be tested for HIV at $t_{1}$, and the remaining partner, $i$, will be tested for HIV at $t_{2}$ if $j$ tests positive for HIV if opportunism is high and if externalities are low (strategic behavior):

$$
\left\{\begin{array}{l}
A_{j}+\Omega_{i}+E_{j} \geq 0 \\
\Omega_{i} \geq 0 \\
A_{i}-\Omega_{i}+E_{i}<0 .
\end{array}\right.
$$

These conditions are satisfied if both partners are likely to be infected and if the HIV test results for $j$ is expected to reflect the serostatus of $i$ (in other words, if $P_{n}$ is high).

Third, only $j$, the partner who is more at risk for HIV infection, undergoes testing for HIV at $t_{1}$, and $i$ refrains from being tested at $t_{2}$ if only $j$ is likely to be infected:

$$
\left\{\begin{array}{l}
A_{i}+E_{i}<0 \\
A_{j}+E_{j} \geq 0 \\
\Omega_{i}<0 .
\end{array}\right.
$$

These conditions are satisfied if $i$ is unlikely to be infected, if $j$ is likely to be infected and if the expected probability $P_{n}$ that the virus was transmitted between $i$ and $j$ is low.

Finally, neither $i$ nor $j$ will take an HIV test if both $i$ and $j$ are unlikely to be infected, that is, if:

$$
\left\{\begin{array}{l}
A_{j}+\Omega_{i}+E_{j}<0 \\
A_{j}+E_{j}<0 .
\end{array}\right.
$$

Proof If both $i$ and $j$ are tested for HIV at $t_{1}$, the expected lifetime utility of the couple is given by:

$$
\begin{aligned}
V^{c}\left(t_{1}^{i}=1, t_{1}^{j}=1\right)= & V^{i}\left(t_{1}^{i}=1, t_{1}^{j}=1\right)+V^{j}\left(t_{1}^{i}=1, t_{1}^{j}=1\right) \\
= & U_{N}+\left[p_{i}+p_{j}\left(1-p_{i}\right) P_{n}\right](H-d)-p_{j}\left(1-p_{i}\right)\left(1-P_{n}\right) p_{k} P_{N} d-C \\
& +U_{N}+\left[p_{j}+p_{i}\left(1-p_{j}\right) P_{n}\right](H-d)-p_{i}\left(1-p_{j}\right)\left(1-P_{n}\right) p_{k} P_{N} d-C .
\end{aligned}
$$

If only $i$ is tested for HIV at $t_{1}$, and $j$ is tested for HIV at $t_{2}$ if $i$ is HIV-positive, then the expected lifetime utility of the couple may be expressed as follows:

$$
\begin{aligned}
V^{c}\left(t_{1}^{i}=1, t_{1}^{j}=0, t_{2}^{j}=1\right)= & V^{i}\left(t_{1}^{i}=1, t_{1}^{j}=0\right)+V^{j}\left(t_{1}^{i}=1, t_{1}^{j}=0, t_{2}^{j}=1\right) \\
= & U_{N}+\left[p_{i}+p_{j}\left(1-p_{i}\right) P_{n}\right](H-d)-p_{j}\left(1-p_{i}\right)\left(1-P_{n}\right) P_{N} d-C \\
& +U_{N}+\left[p_{j} p_{i}+p_{j}\left(1-p_{i}\right) P_{n}+\left(1-p_{j}\right) p_{i} P_{n}\right](H-d-C)-p_{j}\left(1-p_{i}\right)\left(1-P_{n}\right) d \\
& -p_{i}\left(1-p_{j}\right)\left(1-P_{n}\right)\left(p_{k} P_{N} d+C\right) .
\end{aligned}
$$

If only $j$ is tested for HIV at $t_{1}$, and $i$ is tested for HIV at $t_{2}$ if $j$ is HIV-positive, then the expected lifetime utility of the couple is expressed as follows:

$$
\begin{aligned}
V^{c}\left(t_{1}^{i}=0, t_{1}^{j}=1, t_{2}^{i}=1\right)= & V^{i}\left(t_{1}^{i}=0, t_{1}^{j}=1, t_{2}^{i}=1\right)+V^{j}\left(t_{1}^{i}=0, t_{1}^{j}=1\right) \\
= & U_{N}+\left[p_{i} p_{j}+p_{i}\left(1-p_{j}\right) P_{n}+\left(1-p_{i}\right) p_{j} P_{n}\right](H-d-C)-p_{i}\left(1-p_{j}\right)\left(1-P_{n}\right) d \\
& -p_{j}\left(1-p_{i}\right)\left(1-P_{n}\right)\left(p_{j} P_{N} d+C\right) \\
& +U_{N}+\left[p_{j}+p_{i}\left(1-p_{j}\right) P_{n}\right](H-d)-p_{i}\left(1-p_{j}\right)\left(1-P_{n}\right) P_{N} d-C .
\end{aligned}
$$


If $i$ is tested for HIV at $t_{1}$, and $j$ refrains from being tested for HIV at $t_{2}$ (even if $i$ has tested positive for HIV), then the expected lifetime utility of the couple is given by:

$$
\begin{aligned}
V^{c}\left(t_{1}^{i}=1, t_{1}^{j}=0, t_{2}^{j}=0\right)= & V^{i}\left(t_{1}^{i}=1, t_{1}^{j}=0\right)+V^{j}\left(t_{1}^{i}=1, t_{1}^{j}=0, t_{2}^{j}=0\right) \\
= & U_{N}+\left[p_{i}+p_{j}\left(1-p_{i}\right) P_{n}\right](H-d)-p_{j}\left(1-p_{i}\right)\left(1-P_{n}\right) P_{N} d-C \\
& +U_{N}+\left[p_{j} p_{i}+p_{j}\left(1-p_{i}\right) P_{n}+\left(1-p_{j}\right) p_{i} P_{n}\right](-d)-p_{j}\left(1-p_{i}\right)\left(1-P_{n}\right) d \\
& -p_{i}\left(1-p_{j}\right)\left(1-P_{n}\right)\left(p_{k} P_{N} d\right) .
\end{aligned}
$$

If $j$ is tested for HIV at $t_{1}$, and $i$ refrains from being tested for HIV at $t_{2}$ (even if $j$ has tested positive for HIV), then the expected lifetime utility of the couple may be expressed as follows:

$$
\begin{aligned}
V^{c}\left(t_{1}^{i}=0, t_{1}^{j}=1, t_{2}^{i}=0\right)= & V^{i}\left(t_{1}^{i}=0, t_{1}^{j}=1, t_{2}^{i}=0\right)+V^{j}\left(t_{1}^{i}=0, t_{1}^{j}=1\right) \\
= & U_{N}+\left[p_{i} p_{j}+p_{i}\left(1-p_{j}\right) P_{n}+\left(1-p_{i}\right) p_{j} P_{n}\right](-d)-p_{i}\left(1-p_{j}\right)\left(1-P_{n}\right) d \\
& -p_{j}\left(1-p_{i}\right)\left(1-P_{n}\right)\left(p_{k} P_{N} d\right) \\
& +U_{N}+\left[p_{j}+p_{i}\left(1-p_{j}\right) P_{n}\right](H-d)-p_{i}\left(1-p_{j}\right)\left(1-P_{n}\right) P_{N} d-C .
\end{aligned}
$$

If neither member of a couple is tested for HIV infection, then the expected lifetime utility of the couple is given by:

$$
\begin{aligned}
V^{c}\left(t_{1}^{i}=0, t_{1}^{j}=0\right)= & V^{i}\left(t_{1}^{i}=0, t_{1}^{j}=0\right)+V^{j}\left(t_{1}^{i}=0, t_{1}^{j}=0\right) \\
= & U_{N}-d\left[p_{i}+p_{j}\left(1-p_{i}\right)\left(P_{n}+\left(1-P_{n}\right) p_{j} P_{N}\right)\right] \\
& +U_{N}-d\left[p_{j}+p_{i}\left(1-p_{j}\right)\left(P_{n}+\left(1-P_{n}\right) p_{i} P_{N}\right)\right] .
\end{aligned}
$$

If only one of the members of a couple undergoes HIV testing, the partner who has a higher risk of HIV infection will be tested for HIV. Indeed, $V^{c}\left(t_{1}^{i}=0, t_{1}^{j}=1, t_{2}^{i}=1\right)-V^{c}\left(t_{1}^{i}=1, t_{1}^{j}=0, t_{2}^{j}=1\right)=\left(p_{j}-p_{i}\right)\left(1-P_{n}\right)\left(s-c+d\left(1-p_{k}\right) P_{N}\right)$ is greater than 0 if $p_{j} \geq p_{i}$. Similarly, $V^{c}\left(t_{1}^{i}=0, t_{1}^{j}=1, t_{2}^{i}=0\right)-V^{c}\left(t_{1}^{i}=1, t_{1}^{j}=0, t_{2}^{j}=0\right)=\left(p_{j}-p_{i}\right)\left(1-P_{n}\right)(s+$ $\left.d\left(1-p_{k}\right) P_{N}\right)$ if $p_{j} \geq p_{i}$. Therefore, the two cases in which only $i$ undergoes HIV testing are dismissed, and the couple must only compare the remaining four alternatives.

First, both partners will undergo HIV testing at $t_{1}$ if the following three conditions are satisfied: $V^{c}\left(t_{1}^{i}=1, t_{1}^{j}=\right.$ 1) $-V^{c}\left(t_{1}^{i}=0, t_{1}^{j}=1, t_{2}^{i}=1\right)=A_{i}-\Omega_{i}+E_{i} \geq 0, V^{c}\left(t_{1}^{i}=1, t_{1}^{j}=1\right)-V^{c}\left(t_{1}^{i}=0, t_{1}^{j}=1, t_{2}^{i}=0\right)=A_{i}+E_{i} \geq 0$, and $V^{c}\left(t_{1}^{i}=1, t_{1}^{j}=1\right)-V^{c}\left(t_{1}^{i}=0, t_{1}^{j}=0\right)=A_{i}+E_{i}+A_{j}+E_{j} \geq 0$. When $p_{j}>p_{i}$, these three conditions reduce to the system of conditions (B.1).

Second, only the partner who is at greater risk of being infected with HIV, $j$, undergoes HIV testing at $t_{1}$ and his partner, $i$, undergoes testing at $t_{2}$ if the following three conditions are satisfied: $V^{c}\left(t_{1}^{i}=1, t_{1}^{j}=1\right)-V^{c}\left(t_{1}^{i}=\right.$ $\left.0, t_{1}^{j}=1, t_{2}^{i}=1\right)=A_{i}-\Omega_{i}+E_{i}<0, V^{c}\left(t_{1}^{i}=0, t_{1}^{j}=1, t_{2}^{i}=1\right)-V^{c}\left(t_{1}^{i}=0, t_{1}^{j}=1, t_{2}^{i}=0\right)=\Omega_{i} \geq 0$, and $V^{c}\left(t_{1}^{i}=0, t_{1}^{j}=1, t_{2}^{i}=1\right)-V^{c}\left(t_{1}^{i}=0, t_{1}^{j}=0\right)=A_{j}+\Omega_{i}+E_{j} \geq 0$.

Third, the member of the couple who is at higher risk of HIV infection, $j$, undergoes HIV testing at $t_{1}$ but $i$ is not tested at $t_{2}$ if the following three conditions are satisfied: $V^{c}\left(t_{1}^{i}=1, t_{1}^{j}=1\right)-V^{c}\left(t_{1}^{i}=0, t_{1}^{j}=1, t_{2}^{i}=0\right)=A_{i}+E_{i}<0$, $V^{c}\left(t_{1}^{i}=0, t_{1}^{j}=1, t_{2}^{i}=1\right)-V^{c}\left(t_{1}^{i}=0, t_{1}^{j}=1, t_{2}^{i}=0\right)=\Omega_{i}<0$ and $V^{c}\left(t_{1}^{i}=0, t_{1}^{j}=1, t_{2}^{i}=0\right)-V^{c}\left(t_{1}^{i}=0, t_{1}^{j}=0\right)=$ $A_{j}+E_{j} \geq 0$.

Finally, neither $i$ nor $j$ will be tested for HIV if $V^{c}\left(t_{1}^{i}=1, t_{1}^{j}=1\right)-V^{c}\left(t_{1}^{i}=0, t_{1}^{j}=0\right)=A_{i}+E_{i}+A_{j}+E_{j}<0$, $V^{c}\left(t_{1}^{i}=0, t_{1}^{j}=1, t_{2}^{i}=1\right)-V^{c}\left(t_{1}^{i}=0, t_{1}^{j}=0\right)=A_{j}+\Omega_{i}+E_{j}<0$ and $V^{c}\left(t_{1}^{i}=0, t_{1}^{j}=1, t_{2}^{i}=0\right)-V^{c}\left(t_{1}^{i}=0, t_{1}^{j}=0\right)=$ $A_{j}+E_{j}<0$. If $p_{j}>p_{i}$, then these three conditions reduce to the system of conditions (B.4). 
Within this modified framework, the relationship between testing and $P_{n}$ in the hypothesis of couple-based decision making is similar to the relationship that is identified under the hypothesis of individual decision-making. Section 2 revealed that individual $i$ acts strategically and postpones testing if three conditions are satisfied. First, his partner should be expected to test at $t_{1}$, and the results of this test should be observable. Second, $P_{n}$ and opportunism $\Omega$ should be high. In other words, the observation of the partner's test results should be expected to yield significant information about $i$ 's serostatus (serodiscordance should be regarded as unlikely). Third, $A-\Omega$ should be sufficiently low to ensure that $i$ will prefer to postpone testing instead of being tested at $t_{1}$.

If the decision to test is reached in combination with a sexual partner, similar strategic behavior will emerge if these three conditions are satisfied. To observe this similarity, it must first be noted that the externalities $E_{i}$ and $E_{j}$ are decreasing functions of $P_{n}$. Therefore, these positive externalities are sharply reduced after several risky sexual encounters, particularly if the transmission rate of HIV is overestimated.

With this in mind, we can demonstrate that the three aforementioned conditions coincide with the system of conditions (B.2) that identifies strategic behavior in the modified framework for couples-based decisions. The first condition of system (B.2) states that $j$ should be willing to undergo HIV testing: $A_{j}$ represents the expected health benefits from testing, $E_{j}$ is the externality on $i$, and $\Omega_{i}$ represents the gain from the information that is transmitted to $j$. The second condition of system (B.2) states that opportunism $\Omega_{j}$ should be high. This condition is only satisfied if $P_{n}$ is sufficiently high to ensure that the HIV test results for one partner in a couple are expected to be informative about the serostatuses of both members of the couple. Finally, the third condition of system (B.2) reduces to $A_{i}-\Omega_{i}$ if $P_{n}$ is high, as the externality $E_{i}$ vanishes after several risky sexual encounters between $i$ and $j$ if the transmission rate of HIV is overestimated. 